\title{
Software Synthesis and Code Generation for Signal Processing Systems *
}

\author{
Shuvra S. Bhattacharyya \\ University of Maryland \\ Department of Electrical and Computer Engineering \\ College Park, MD 20742, USA
}

\author{
Rainer Leupers, Peter Marwedel \\ University of Dortmund \\ Department of Computer Science 12 \\ 44221 Dortmund, Germany
}

\begin{abstract}
The role of software is becoming increasingly important in the implementation of DSP applications. As this trend intensifies, and the complexity of applications escalates, we are seeing an increased need for automated tools to aid in the development of DSP software. This paper reviews the state of the art in programming language and compiler technology for DSP software implementation. In particular, we review techniques for high level, block-diagram-based modeling of DSP applications; the translation of block diagram specifications into efficient $\mathrm{C}$ programs using global, target-independent optimization techniques; and the compilation of C programs into streamlined machine code for programmable DSP processors, using architecture-specific and retargetable back-end optimizations. In our review, we also point out some important directions for further investigation.
\end{abstract}

\section{Introduction}

Although dedicated hardware can provide significant speed and power consumption advantages for signal processing applications [1], extensive programmability is becoming an increasingly desirable feature of implementation platforms for VLSI signal processing. The trend towards programmable platforms is fueled by tight time-to-market windows, which in turn result from intense competition among DSP product vendors, and from the rapid evolution of technology, which shrinks the life cycle of consumer products. As a result of short time-to-market windows, designers are often forced to begin architecture design and system implementation before the specification of a product is fully completed. For example, a portable communication product is often designed before the signal transmission standards under which it will operate are finalized, or before the full range of standards that will be supported by the product is agreed upon. In such an environment, late changes in the design cycle are mandatory. The need to quickly make such late changes requires the use of software. Furthermore, whether or not the product specification is fixed beforehand, software-based implementations using off-the-shelf processors take significantly less verification effort compared to custom hardware solutions.

Although the flexibility offered by software is critical in DSP applications, the implementation of production quality DSP software is an extremely complex task. The complexity arises from the diversity of critical constraints that must be satisfied; typically these constraints involve stringent requirements on metrics such as latency, throughput, power consumption, code size, and data storage requirements. Additional constraints include the need to ensure key implementation properties such as

*Technical report UMIACS-TR-99-57, Institute for Advanced Computer Studies, University of Maryland, College Park, 20742, September, 1999. S. S. Bhattacharyya was supported in this work by the US National Science Foundation (CAREER, MIP9734275) and Northrop Grumman Corp. R. Leupers and P. Marwedel were supported by HP EESof, California. 
bounded memory requirements and deadlock-free operation. As a result, unlike developers of software for general-purpose platforms, DSP software developers routinely engage in meticulous tuning and simulation of program code at the assembly language level.

Important industry-wide trends at both the programming language level and the processor architecture level have had a significant impact on the complexity of DSP software development. At the architectural level, a specialized class of microprocessors has evolved that is streamlined to the needs of DSP applications. These DSP-oriented processors, called programmable digital signal processors (PDSPs), employ a variety of special-purpose architectural features that support common DSP operations such as digital filtering, and fast Fourier transforms [2, 3, 4]. At the same time, they often exclude features of general purpose processors, such as extensive memory management support, that are not important for many DSP applications.

Due to various architectural irregularities in PDSPs, which are required for their exceptional cost/performance and power/performance trade-offs [2], compiler techniques for general-purpose processors have proven to be inadequate for exploiting the power of PDSP architectures from high level languages [5]. As a result, the code quality of high-level procedural language (such as C) compilers for PDSPs has been several hundreds of percent worse than manually-written assembly language code $[6,52]$. This situation has necessitated the widespread use of assembly-language coding, and tedious performance tuning, in DSP software development. However, in recent years, a significant research community has evolved that is centered around the development of compiler technology for PDSPs. This community has begun to narrow the gap between compiler-generated code and manually optimized code.

It is expected that innovative processor-specific compilation techniques for PDSPs will provide a significant productivity boost in DSP software development, since such techniques will us allow to take the step from assembly programming of PDSPs to the use of high-level programming languages. The key approach to reduce the overhead of compiler-generated code is the development of DSP-specific compiler optimization techniques. While classical compiler technology is often based on the assumption of a regular processor architecture, DSP-specific techniques are designed to be capable of exploiting the special architectural features of PDSPs. These include special purpose registers in the data path, dedicated memory address generation units, and a moderate degree of instruction-level parallelism.

To illustrate this, consider the architecture of a popular fixed-point DSP (TI TMS320C25) in fig. 1. Its data path comprises the registers TR, PR, and ACCU, each of which plays a specific role in communicating values between the functional units of the processor. This structure allows for a very efficient implementation of DSP algorithms (e.g. filtering algorithms). More regular architectures (e.g. with general-purpose registers) would, for instance, require more instruction bits for addressing the registers and more power for reading and writing the register file.

From a compiler viewpoint, the mapping of operations, program variables, and intermediate results to the data path in fig. 1 must be done in such a way, that the amount of data transfer instructions between the registers is minimized. The address generation unit (AGU) comprises a special ALU and is capable of performing address arithmetic in parallel to the central data path. In particular, it provides parallel auto-increment instructions for address registers. As we will show later, exploitation of this feature in a compiler demands for an appropriate memory layout of program variables. Besides the AGU, also the data path offers a certain degree of instruction-level parallelism. For instance, loading a memory value into register TR and accumulating a product stored in PR can be performed in parallel within a single machine instruction. Since such parallelism cannot be explicitly described in programming languages like $\mathrm{C}$, compilers need to carefully schedule the generated machine instructions, so as to exploit the potential parallelism and to generate fast and dense code.

Further architectural features frequently present in PDSPs include parallel memory banks (providing higher memory access bandwidth), chained operations (such as multiply-accumulate), special arithmetic operations (such as addition with saturation), and mode registers (for switching between different arithmetic modes).

For most of the architectural features mentioned above, dedicated code optimization techniques have been developed recently, an overview of which will be given in section 3. Many of these optimizations are computationally complex, resulting 
in a comparatively low compilation speed. This is intensified by the fact that compilers for PDSPs, besides the need for specific optimization techniques, have to deal with the phase coupling problem. The compilation process is traditionally divided into the phases of code selection, register allocation, and instruction scheduling, which have to be executed in a certain order. For all possible phase orders, the approach of separate compilation phases results in a code quality overhead, since each phase may impose obstructing constraints on subsequent phases, which would not have been necessary from a global viewpoint. While for regular processor architectures like RISCs this overhead is moderate and thus tolerable, it is typically much higher for irregular processor architectures as found in PDSPs. Therefore, it is desirable to perform the compilation phases in a coupled fashion, where the different phases mutually exchange information so as to achieve a global optimum.

Even though phase-coupled compiler techniques lead to a further increase in compilation time, it is widely agreed in the DSP software developer community that high compilation speed is of much lower concern than high code quality. Thus, compilation times of minutes or even hours may be perfectly acceptable in many cases. This fact gives good opportunities for novel computation-intensive approaches to compiling high level languages for PDSPs, which however would not be acceptable in general-purpose computing.

Besides pure code optimization issues, the large variety of PDSPs (both standard "off-the-shelf" processors and application specific processors) currently in use create a problem of economic feasibility of compiler construction. Since code optimization techniques for PDSPs are highly architecture-specific by nature, a huge amount of different optimization techniques were required to build efficient compilers for all PDSPs available on the market. Therefore, in this paper we will also briefly discuss techniques for retargetable compilation. Retargetable compilers are capable of generating code not only for a single target processor but for a class of processors, thereby reducing the number of compilers required. This is achieved by providing the compiler with a description of the machine for which code is to be generated, instead of hard-coding the machine description in the compiler. We will mention different approaches of processor modeling for retargetable compilation. Retargetability permits to quickly generate compilers for new processors. If the processor description formalism is flexible enough, then retargetable compilers may also assist in customizing an only partially predefined processor architecture for a given application.

At the system specification level, the past several years have seen increased use of block-diagram based, graphical programming environments for digital signal processing. Such graphical programming environments, which enable DSP systems to be specified as hierarchies of block diagrams, offer several important advantages. Perhaps the most obvious of these advantages is their intuitive appeal. Although visual programming languages have seen limited use in many application domains, DSP system designers are used to thinking of systems in terms of graphical abstractions, such as signal flow diagrams, and thus, block diagram specification via a graphical user interface is a convenient and natural programming interface for DSP design tools.

An illustration of a block diagram DSP system, developed using the Ptolemy design environment [7], is shown in fig. 2. This is an implementation of a discrete wavelet transform [8] application. The top part of the figure shows the highest level of the block diagram specification hierarchy. Many of the blocks in the specification are hierarchical, which means that the internal functionality of the blocks are also specified as block diagrams ("nested" block diagrams). Blocks at the lowest level of the specification hierarchy, such as the individual FIR filters, are specified in a meta-C language ( $\mathrm{C}$ augmented with special constructs for specifying block parameters and interface information).

In addition to offering intuitive appeal, the specification of systems in terms of connections between pre-defined, encapsulated functional blocks naturally promotes desirable software engineering practices such as modularity and code reuse. As the complexity of applications increases continually while time-to-market pressures remain intense, reuse of design effort across multiple products is becoming more and more crucial to meeting development schedules.

In addition to their syntactic and software engineering appeal, there are a number of more technical advantages of graphical DSP tools. These advantages hinge on the use of appropriate models of computation to provide the precise underlying 
block diagram semantics. In particular, the use of dataflow models of computation can enable the application of powerful verification and synthesis techniques. Broadly speaking, dataflow modeling involves representing an application as a directed graph in which the graph vertices represent computations and edges represent logical communication channels between computations. Dataflow-based graphical specification formats are used widely in commercial DSP design tools such as COSSAP by Synopsys, the Signal Processing Worksystem by Cadence, and the Advanced Design System by Hewlett-Packard. These three commercial tools all employ the synchronous dataflow model [9], the most popular variant of dataflow in existing DSP design tools. Synchronous dataflow specification allows bounded memory determination and deadlock detection to be performed comprehensively and efficiently at compile time. In contrast, both of these verification problems are in general impossible to solve (in finite time) for general purpose programming languages such as $\mathrm{C}$.

Potentially the most useful benefit of dataflow-based graphical programming environments for DSP is that carefullyspecified graphical programs can expose coarse-grain structure of the underlying algorithm, and this structure can be exploited to improve the quality of synthesized implementations in a wide variety of ways. For example, the process of scheduling determining the order in which the computations in an application will execute - typically has a large impact on all of the key implementation metrics of a DSP system. A dataflow-based system specification exposes high-level scheduling flexibility that is often not possible to deduce manually or automatically from an assembly language or high-level procedural language specification. This scheduling flexibility can be exploited by a synthesis tool to streamline an implementation based on the given set of performance and cost constraints. We will elaborate on dataflow-based scheduling in sections 2.1.2 and 2.2.

Although graphical dataflow-based programming tools for DSP have become increasingly popular in recent years, the use of these tools in industry is largely limited to simulation and prototyping. The quality of today's graphical programming tools is not sufficient to consistently deliver production-quality implementations. As with procedural language compilation technology for PDSPs, synthesis from dataflow-based graphical specifications offers significant promise for the future, and is an important challenge confronting the DSP design and implementation research community today. Furthermore, these two forms of compiler technology are fully complementary to one another: the mixture of dataflow and $\mathrm{C}$ (or any other procedural language), as described in the example of fig. 2, is an especially attractive specification format. In this format, coarse-grain "subprogram" interactions are specified in dataflow, while the functionality of individual subprograms is specified in C. Thus, dataflow synthesis techniques optimize the final implementation at the inter-subprogram level, while C compiler technology is required to perform fine-grained optimization within subprograms.

This paper motivates the problem of compiler technology development for DSP software implementation, provides a tutorial overview of modeling and optimization issues that are involved in the compilation of DSP software, and provides a review of techniques that have been developed by various researchers to address some of these issues. The first part of our overview focuses on coarse-grain software modeling and optimization issues pertinent to the compilation of graphical dataflow programs, and the second part focuses on fine-grained issues that arise in the compilation of high level procedural languages such as $\mathrm{C}$.

These two levels of compiler technology (coarse-grain and fine grain) are commonly referred to as software synthesis and code generation, respectively. More specifically, by software synthesis, we mean the automated derivation of a software implementation (application program) in some programming language given a library of subprogram modules, a subset of selected modules from this library, and a specification of how these selected modules interact to implement the target application. Fig. 2 is an example of a program specification that is suitable for software synthesis. Here, synchronous dataflow semantics are used to specify subprogram interactions. In section 2.2, we explore software synthesis issues for DSP.

On the other hand, code generation refers to the mapping of a software implementation in some programming language to an equivalent machine program for a specific programmable processor. Thus, the mapping of a $\mathrm{C}$ program on to the specific resources of the datapath in fig. 1 is an example of code generation. We explore DSP code generation technology in section 3. 


\section{Compilation of dataflow programs to application programs}

\subsection{Dataflow modeling of DSP systems}

To perform simulation, formal verification, or any kind of compilation from block-diagram DSP specifications, a precise set of semantics is needed that defines the interactions between different computational blocks in a specification. Dataflow-based computational models have proven to provide block-diagram semantics that are both intuitive to DSP system designers, and efficient from the point of view of verification and synthesis.

In the dataflow paradigm, a computational specification is represented as a directed graph. Vertices in the graph (called actors) correspond to the computational modules in the specification. In most dataflow-based DSP design environments, actors can be of arbitrary complexity. Typically, they range from elementary operations such as addition or multiplication to DSP subsystems such as FFT units or adaptive filters.

An edge $\left(v_{1}, v_{2}\right)$ in a dataflow graph represents the communication of data from $v_{1}$ to $v_{2}$. More specifically, an edge represents a FIFO (first-in-first-out) queue that buffers data samples (tokens) as they pass from the output of one actor to the input of another. If $e=\left(v_{1}, v_{2}\right)$ is a dataflow edge, we write $\operatorname{src}(e)=v_{1}$, and $\operatorname{snk}(e)=v_{2}$. When dataflow graphs are used to represent signal processing applications, a dataflow edge $e$ has a non-negative integer delay $\operatorname{del}(e)$ associated with it. The delay of an edge gives the number of initial data values that are queued on the edge. Each unit of dataflow delay is functionally equivalent to the $z^{-1}$ operator: the sequence of data values $\left\{y_{n}\right\}$ generated at the input of the actor $\operatorname{snk}(e)$ is equal to the the shifted sequence $\left\{x_{n-\operatorname{del}(e)}\right\}$, where $\left\{x_{n}\right\}$ is the data sequence generated at the output of the actor $\operatorname{src}(e)$.

\subsubsection{Consistency}

Under the dataflow model, an actor can execute at any time that it has sufficient data on all input edges. An attempt to execute an actor when this constraint is not satisfied is said to cause buffer underflow on all edges that do not contain sufficient data. For dataflow modeling to be useful for DSP systems, the execution of actors must also accommodate input data sequences of unbounded length. This is because DSP applications often involve operations that are applied repeatedly to samples in indefinitely long input signals. For an implementation of a dataflow specification to be practical, the execution of actors must be such that the number of tokens queued on each FIFO buffer (dataflow edge) must remain bounded throughout the execution of the dataflow graph. In other words, there should not be unbounded data accumulation on any edge in the dataflow graph.

In summary, executing a dataflow specification of a DSP system involves two fundamental, processor-independent requirements - avoiding buffer underflow and avoiding unbounded data accumulation (buffering). The dataflow model imposes no further constraints on the sequence in which computations (actors) are executed. On the other hand, in procedural languages, such as $\mathrm{C}$ and FORTRAN, the ordering of statements as well as the use of control-flow constructs imply sequencing constraints beyond those that are required to satisfy data dependencies. By avoiding the overspecification of execution ordering, dataflow specifications provide synthesis tools with full flexibility to streamline the execution order to match the relevant implementation constraints and optimization objectives. This feature of dataflow is of critical importance for DSP implementation since, as we will see throughout the rest of this section, the execution order has a large impact on most important implementation metrics, such as performance, memory requirements, and power consumption.

The term "consistency" refers to the two essential requirements of DSP dataflow specifications - the absence of overflow and unbounded data accumulation. We say that a consistent dataflow specification is one that can be implemented without any chance of buffer underflow or unbounded data accumulation (regardless of the input sequences that are applied to the system). If there exist one or more sets of input sequences for which underflow and unbounded buffering are avoided, and there also exist one or more sets for which underflow or unbounded buffering results, we say that a specification is partially consistent. A dataflow specification that is neither consistent nor partially consistent is called an inconsistent specification. More elaborate forms of consistency based on a probabilistic interpretation of token flow are explored in [10]. 
Clearly, consistency is a highly desirable property for DSP software implementation. For most consistent dataflow graphs, tight bounds can be derived on the numbers of data values that coexist (data that has been produced but not yet consumed) on the individual edges (buffers). For such graphs, all buffer memory allocation can be performed statically, and thus, the overhead of dynamic memory allocation can be avoided entirely. This is a valuable feature when attempting to derive a streamlined software implementation.

\subsubsection{Scheduling}

A fundamental task in synthesizing software from an SDF specification is that of scheduling, which refers to the process of determining the order in which the actors will be executed. Scheduling is either dynamic or static. In static scheduling, the actor execution order is specified at synthesis time, and is fixed - in particular, the order is not data-dependent. To be useful in handling indefinitely long input data sequences, a static schedule must be periodic. A periodic, static schedule can be implemented in a finite amount of program memory space by encapsulating the program code for one period of the schedule within an infinite loop. Indeed, this is how such schedules are most often implemented in practice.

In dynamic scheduling, the sequence of actor executions (schedule) is not specified during synthesis, and run-time decision-making is required to ensure that actors are executed only when their respective input edges have sufficient data. Disadvantages of dynamic scheduling include the overhead (execution time and power consumption) of performing scheduling decisions at run-time, and decreased predictability, especially in determining whether or not any relevant real-time constraints will be satisfied. However, if the data production/consumption behavior of individual actors exhibits significant datadependence, then dynamic scheduling may be required to avoid buffer underflow and unbounded data accumulation. Furthermore, if the performance characteristics of actors are impossible to estimate accurately, then effective dynamic scheduling leads to better performance by adaptively streamlining the schedule evolution to match the dynamic characteristics of the actors.

For most DSP applications, including the vast majority of applications that are amenable to the SDF model mentioned in section 1, actor behavior is highly predictable. For such applications, given the tight cost and power constraints that are typical of embedded DSP applications, it is highly desirable to avoid dynamic scheduling overhead as much as possible. The ultimate goal under such a high level of predictability is a (periodic) static schedule. If it is not possible to construct a static schedule, then it is desirable to identify "maximal" subsystems that can be scheduled statically, and use a small amount of dynamic decision-making to coordinate the execution of these statically-scheduled subsystems. Schedules that are constructed using such a hybrid, mostly static approach are called quasi-static schedules.

\subsubsection{Synchronous dataflow}

A dataflow computation model can be viewed as a subclass of dataflow graph specifications. A wide variety of dataflow computational models can be conceived depending on restrictions that are imposed on the manner in which dataflow actors consume and produce data. For example, synchronous dataflow (SDF), which is the simplest and currently the most popular form of dataflow for DSP, imposes the restriction that the number of data values produced by an actor onto each output edge is constant, and similarly the number of data values consumed by an actor from each input edge is constant. Thus, an SDF edge $e$ has two additional attributes - the number of data values produced onto $e$ by each invocation of the source actor, denoted $\operatorname{prd}(e)$, and the number of data values consumed from $e$ by each invocation of the sink actor, denoted cns $(e)$.

The example shown in fig. 2 conforms to the SDF model. An SDF abstraction of a scaled-down and simplified version of this system is shown in fig. 3. Here each edge is annotated with the number of data values produced and consumed by the source and sink actors, respectively. For example, $\operatorname{prd}((B, C))=1$, and $\operatorname{cns}((B, C))=2$.

The restrictions imposed by the SDF model offer a number of important advantages.

- Simplicity. Intuitively, when compared to more general types of dataflow actors, actors that produce and consume 
data in constant-sized packets are easier to understand, develop, interface to other actors, and maintain. This property is difficult to quantify; however, the rapid and extensive adoption of SDF in DSP design tools clearly indicates that designers can easily learn to think of functional specifications in terms of the SDF model.

- Static scheduling and memory allocation. For SDF graphs, there is no need to resort to dynamic scheduling, or even quasi-static scheduling. For a consistent SDF graph, underflow and unbounded data accumulation can always be avoided with a periodic, static schedule. Moreover, tight bounds on buffer occupancy can be computed efficiently. By avoiding the run-time overheads associated with dynamic scheduling and dynamic memory allocation, efficient SDF graph implementations offer significant advantages when cost, power, or performance constraints are severe.

- Consistency verification. A dataflow model of computation is a decidable dataflow model if it can be determined in finite time whether or not an arbitrary specification in the model is consistent. We say that a dataflow model is a binaryconsistency model if every specification in the model is either consistent or inconsistent. In other words, a model is a binary-consistency model if it contains no partially consistent specifications. All of the decidable dataflow models that are used in practice today are binary-consistency models.

Binary consistency is convenient from a verification point of view since consistency becomes an inherent property of a specification: whether or not buffer underflow or unbounded data accumulation arises is not dependent on the input sequences that are applied. Of course, such convenience comes at the expense of restricted applicability. A binary-consistency model cannot be used to specify all applications.

The SDF model is a binary-consistency model, and efficient verification techniques exist for determining whether or not an SDF graph is consistent. Although SDF has limited expressive power in exchange for this verification efficiency, the model has proven to be of great practical value. SDF encompasses a broad and important class of signal processing and digital communications applications, including modems, multirate filter banks [8], and satellite receiver systems, just to name a few $[9,11,12]$.

For SDF graphs, the mechanics of consistency verification are closely related to the mechanics of scheduling. The interrelated problems of verifying and scheduling SDF graphs are discussed in detail below.

\subsubsection{Static scheduling of SDF graphs}

The first step in constructing a static schedule for an SDF graph $G=(V, E)$ is determining the number of times $i(A)$ that each actor $A \in V$ should be invoked in one period of the schedule. To ensure that the schedule period can be repeated indefinitely without unbounded data accumulation, the constraint

$$
\begin{array}{r}
i(\operatorname{src}(e)) \operatorname{prd}(e)=i(\operatorname{snk}(e)) \operatorname{cns}(e), \\
\text { for every edge } e \in E
\end{array}
$$

must be satisfied. The system of equations (1) is called the set of balance equations for $G$.

Clearly, a useful periodic schedule can be constructed only if the balance equations have a positive integer solution $i^{*}$ $\left(i^{*}(A)>0\right.$ for all $\left.A \in V\right)$. Lee and Messerschmitt have shown that for a general SDF graph $G$, exactly one of the following conditions holds [9]:

- The zero vector is the only solution to the balance equations, or

- There exists a minimal positive integer solution $q$ to the balance equations, and thus every positive integer solution $i^{\prime}$ satisfies $i^{\prime}(A) \geq q(A)$ for all $A$. This minimal vector $q$ is called the repetitions vector of $G$. 
If the former condition holds, then $G$ is inconsistent. Otherwise, a bounded buffer periodic schedule can be constructed provided that it is possible to construct a sequence of actor executions such that buffer underflow is avoided, and each actor $A$ is executed exactly $q(A)$ times. Given a consistent SDF graph, we refer to an execution sequence that satisfies these two properties as a valid schedule period, or simply a valid schedule. Clearly, a bounded memory static schedule can be implemented in software by encapsulating the implementation of any valid schedule within an infinite loop.

A linear-time $(O(|V|+|E|))$ algorithm to determine whether or not a repetitions vector exists, and to compute a repetitions vector whenever one does exist can be found in [11].

For example, consider the SDF graph shown in fig. 3. The repetitions vector components for this graph are given by

$$
\begin{aligned}
& q(A)=q(B)=q(P)=q(Q)=4 \\
& q(C)=q(D)=q(E)=q(H)=q(M)=q(N)=q(O)=2 \\
& q(F)=q(G)=q(I)=q(J)=q(K)=q(L)=1
\end{aligned}
$$

If a repetitions vector exists for an SDF graph, but a valid schedule does not exist, then the graph is said to be deadlocked. Thus, an SDF graph is consistent if and only if a repetitions vector exists, and the graph is not deadlocked. In general, whether or not a graph is deadlocked depends on the edge delays $\{\operatorname{del}(e) \mid e \in E\}$ as well the production and consumption parameters $\{\operatorname{src}(e)\}$ and $\{\operatorname{snk}(e)\}$. An example of a deadlocked SDF graph is given in fig. 4. An annotation of the form $n \mathrm{D}$ next to an edge in the figure represents a delay of $n$ units. Note that the repetitions vector for this graph is given by

$$
q(A)=3, q(B)=2, q(C)=1 .
$$

Once a repetitions vector $q$ has been computed, deadlock detection and the construction of a valid schedule can be performed concurrently. Premature termination of the scheduling procedure - termination before each actor $A$ has been fully scheduled (scheduled $q(A)$ times) - indicates deadlock. One simple approach is to schedule actor invocations one at a time and simulate the buffer activity in the dataflow graph accordingly until all actors are fully scheduled. The buffer simulation is necessary to ensure that buffer overflow is avoided. A pseudocode specification of this simple approach can be found in [11]. Lee and Messerschmitt show that this approach terminates prematurely if and only if the input graph is deadlocked, and otherwise, regardless of the specific order in which actors are selected for scheduling, a valid schedule is always constructed [13].

In summary, SDF is currently the most widely-used dataflow model in commercial and research-oriented DSP design tools. Commercial tools that employ SDF semantics include Simulink by The Math Works, SPW by Cadence, and HP Ptolemy by Hewlett Packard. SDF-based research tools include Gabriel [14] and several key domains in Ptolemy [7], from from U.C. Berkeley; and ASSIGN from Carnegie Mellon [15]. The SDF model offers efficient verification of consistency for arbitrary specifications, and efficient construction of static schedules for all consistent specifications. Our discussion above outlined a simple, systematic technique for constructing a static schedule whenever one exists. In practice, however, it is preferable to employ more intricate scheduling strategies that take careful account of the costs (performance, memory consumption, etc.) of the generated schedules. In section 2.2, we will discuss techniques for streamlined scheduling of SDF graphs based on the constraints and optimization objectives of the targeted implementation. In the remainder of this section, we discuss a number of useful extensions to the SDF model.

\subsubsection{Cyclo-static dataflow}

Cyclo-static dataflow (CSDF) and scalable synchronous dataflow (described in section 2.1.6) are presently the most widelyused extensions of SDF. In CSDF, the number of tokens produced and consumed by an actor is allowed to vary as long the 
variation takes the form of a fixed, periodic pattern $[16,17]$. More precisely, each actor A in a CSDF graph has associated with it a fundamental period $\tau(A) \in\{1,2, \ldots\}$, which specifies the number of phases in one minimal period of the cyclic production/consumption pattern of $A$. For each input edge $e$ to $A$, the scalar SDF attribute $\operatorname{cns}(e)$ is replaced by a $\tau(A)$-tuple $C_{e, 1}, C_{e, 2}, \ldots, C_{e, \tau(A)}$, where each $C_{e, i}$ is a nonnegative integer that gives the number of data values consumed from $e$ by $A$ in the $i$ th phase of each period of $A$. Similarly, for each output edge $e, \operatorname{prd}(e)$ is replaced by a $\tau(A)$-tuple $P_{e, 1}, P_{e, 2}, \ldots, P_{e, \tau(A)}$, which gives the numbers of data values produced in successive phases of $A$.

A simple example of a CSDF actor is illustrated in fig. 5(a). This actor is a conventional downsampler actor (with downsampling factor 3) from multirate signal processing. Functionally, a downsampler, performs the function $y[i]=x[N(i-$ $1)+1]$, where for $k=1,2, \ldots, y[k]$ and $x[k]$ denote the $k$ data values produced and consumed, respectively. Thus, for every input value that is copied to the output, $N-1$ input values are discarded. As shown in fig. 5(b) for $n=3$, this functionality can be specified by a CSDF actor that has $N$ phases. A data value is consumed on the input for all $N$ phases, resulting in the $N$-component consumption tuple $(1,1, \ldots, 1)$; however, a data value is produced onto the output edge only on the first phase, resulting in the production tuple $(1,0,0, \ldots, 0)$.

Like SDF, CSDF is a binary consistency model, and it is possible to perform efficient verification of bounded memory requirements and buffer underflow avoidance for CSDF graphs [17]. Furthermore, static schedules can always be constructed for consistent CSDF graphs.

A CSDF actor $A$ can easily be converted into an SDF actor $A^{\prime}$ such that if identical sequences of input data values are applied to $A$ and $A^{\prime}$, then identical output data sequences result. Such a functionally equivalent SDF actor $A^{\prime}$ can be derived by having each invocation of $A^{\prime}$ implement one fundamental CSDF period of $A$ (that is, $\tau(A)$ successive phases of $A$ ). Thus, for each input edge $e^{\prime}$ of $A^{\prime}$, the SDF parameters of $e^{\prime}$ are given by

- $\operatorname{del}\left(e^{\prime}\right)=\operatorname{del}(e)$

- $\operatorname{prd}\left(e^{\prime}\right)=\sum_{i=1}^{\tau(A)} P_{e, i}$, and

- $\operatorname{cns}\left(e^{\prime}\right)=\sum_{i=1}^{\tau(A)} C_{e, i}$,

where $e$ is the corresponding input edge to the CSDF actor $A$. Applying this conversion to the downsampler example discussed above gives an "SDF equivalent" downsampler that consumes a block of $N$ input data values on each invocation, and produces a single data value, which is a copy of the first value in the input block. The SDF equivalent for fig. 5(a) is illustrated in fig. 5(b).

Since any CSDF actor can be converted to a functionally equivalent SDF actor, it follows that CSDF does not offer increased expressive power at the level of individual actor functionality (input-output mappings). However, the CSDF model can offer increased flexibility in compactly and efficiently representing interactions between actors.

As an example of increased flexibility in expressing actor interactions, consider the CSDF specification illustrated in fig. 6. This specification represents a recursive digital filter computation of the form

$$
y_{n}=k^{2} y_{n-1}+k x_{n}+x n-1 .
$$

In fig. 6, the two-phase CSDF actor labeled $A$ represents a scaling (multiplication) by the constant factor $k$. In each of its two phases, actor $A$ consumes a data value from one of its input edges, multiplies the data value by $k$, and produces the resulting value onto one of its output edges. The CSDF specification of fig. 6 thus exploits our ability to compute (4) using the equivalent formulation

$$
y_{n}=k\left(k y_{n-1}+x_{n}\right)+x n-1,
$$

which requires only addition blocks and $k$-scaling blocks. Furthermore, the two $k$-scaling operations contained in ( 5) are consolidated into a single CSDF actor (actor $A$ ). 
Such consolidation of distinct operations from different data streams offers two advantages. First, it leads to more compact representations since fewer vertices are required in the CSDF graph. For large or complex applications, this can result in more intuitive representations, and can reduce the time required to perform various analysis and synthesis tasks. Second, it allows a precise modeling of resource sharing decisions - pre-specified bindings of multiple operations in a DSP application onto individual hardware resources (such as functional units) or software resources (such as subprograms) within the framework of dataflow. Such pre-specified bindings may arise from constraints imposed by the designer, and from decisions taken during synthesis or design space exploration.

The ability to compactly and precisely model the sharing of actors in CSDF stems from the ability to selectively "turn off" data dependencies from arbitrary subsets of input edges in any given phase of an actor. In contrast, an SDF actor requires at least one data value on each input edge before it can be invoked. In the presence of feedback loops, this requirement may preclude a shared representation of an actor in SDF, even though it may be possible to achieve the desired sharing using a functionally equivalent CSDF actor. This is illustrated in fig. 7, which is derived from the CSDF specification of fig. 6 by replacing the "shared" CSDF actor with its functionally equivalent SDF counterpart. Since the graph of fig. 7 contains a delay-free cycle, clearly we can conclude that the graph is deadlocked, and thus a valid schedule does not exist. In other words, this is an inconsistent dataflow specification. In contrast, it is easily verified that the schedule $A_{1} F D B A_{2} C E G$ is a valid schedule for the CSDF specification of fig. 6, where $A_{1}$ and $A_{2}$ denote the first and second phases of the CSDF actor $A$, respectively.

Similarly, an SDF model of a hierarchical actor may introduce deadlock in a system specification, and such deadlock can often be avoided by replacing the hierarchical SDF actor with a functionally equivalent hierarchical CSDF actor. Here, by a hierarchical SDF actor we mean an actor whose internal functionality is specified by an SDF graph. The utility of CSDF in constructing hierarchical specifications is illustrated in fig. 8.

CSDF also offers decreased buffering requirements for some applications. An illustration is shown in fig. 9. Fig. 9(a) depicts a system in which $N$-element blocks of data are alternately distributed from the data source to two processing modules $M_{1}$ and $M_{2}$. The actor that performs the distribution is modeled as a two-phase CSDF actor that inputs an $N$-element data block on each phase, sends the input block to $M_{1}$ in the first phase, and sends the input block to $M_{2}$ in the second phase. It is easily seen that the CSDF specification of fig. 9(a) can be implemented with a buffer of size $N$ on each of the three edges. Thus, the total buffering requirement is $3 N$ for this specification.

If we replace the CSDF "block-distributor" actor with its functionally equivalent SDF counterpart, then we obtain the pure SDF specification depicted in fig. 9(b). The SDF version of the distributor must process two blocks at a time to conform to SDF semantics. As a result, the edge that connects the data source to the distributor requires a buffer of size $2 N$. Thus, the total buffering requirement of the SDF graph of fig. 9(b) is $4 N$, which is 33\% greater than the CSDF version of fig. 9(a).

Yet another advantage offered by CSDF is that by decomposing actors into a finer level (phase-level) of specification granularity, basic behavioral optimizations such as constant propagation and dead code elimination [18, 54] are facilitated significantly [19]. As a simple example of dead code elimination with CSDF, consider the CSDF specification shown in fig. 10(a) of a multirate FIR filtering system that is expressed in terms of basic multirate building blocks. From this graph, the equivalent expanded homogeneous SDF graph, shown in fig. 10(b), can be derived using concepts discussed in $[9,17]$. In the expanded graph, each actor corresponds to a single phase of a CSDF actor or a single invocation of an SDF actor within a single period of a periodic schedule. From fig. 10(b) it is apparent that the results of some computations (SDF invocations or CSDF phases) are never needed in the production of any of the system outputs. Such computations correspond to dead code and can be eliminated during synthesis without compromising correctness. For this example, the complete set of subgraphs that correspond to dead code is illustrated in fig. 10(b). Parks, Pino, and Lee show that such "dead subgraphs" can be detected with a straightforward algorithm [19].

In summary, CSDF is a useful generalization of SDF that maintains the properties of binary consistency, efficient verification, and static scheduling while offering a more rich range of inter-actor communication patterns, improved support for 
hierarchical specifications, more economical data buffering, and improved support for basic behavioral optimizations. CSDF concepts are used in a number of commercial design tools such as DSP Canvas by Angeles Design Systems, and Virtuoso Synchro by Eonic Systems.

\subsubsection{Scalable synchronous dataflow}

The scalable synchronous dataflow (SSDF) model is an extension of SDF that enables software synthesis of vectorized implementations, which exploit the facility for efficient block processing in many DSP applications [20]. The internal (host language) specification of an SSDF actor $A$ assumes that the actor will be executed in groups of $N_{v}(A)$ successive invocations, which operate on $\left(N_{v}(A) c n s(e)\right)$-unit blocks of data at a time from each input edge $e$. Such block processing reduces the rate of inter-actor context switching, and context switching between successive code segments within complex actors, and it also may improve execution efficiency significantly on deeply pipelined architectures. The vectorization parameter $N_{v}$ of each SSDF actor is selected carefully during synthesis. This selection should be based on constraints imposed by the SSDF graph structure; the memory constraints and performance requirements of the target application; and on the following extended version of the SDF balance equation (1) constraints

$$
N_{v}(\operatorname{src}(e)) q(\operatorname{src}(e)) \operatorname{prd}(e)=N_{v}(\operatorname{snk}(e)) q(\operatorname{snk}(e)) \operatorname{cns}(e)
$$$$
\text { for every edge } e \text { in the SSDF graph, }
$$

where $q$ is the repetitions vector of the SDF graph that results when the vectorization parameter of each actor is set to unity. Since the utility of SSDF is closely tied to optimized synthesis techniques, we defer detailed discussion of SSDF to section 2.2.4, which focuses on throughput-oriented optimization issues for software synthesis.

SSDF is a key specification model in the popular COSSAP design tool that was originally developed by Cadis and the Aachen University of Technology [21], and is now developed by Synopsys.

\subsubsection{Other dataflow models}

The SDF, CSDF, and SSDF models discussed above are all used in widely-distributed DSP design tools. A number of more experimental DSP dataflow models have also been proposed in recent years. Although these models all offer additional insight on dataflow modeling for DSP, further research and development is required before the practical utility of these models is clearly understood. In the remainder of this section, we briefly review some of these experimental models.

The multidimensional synchronous dataflow model (MDSDF), proposed by Lee [22], and explored further by Murthy [23], extends SDF concepts to applications that operate on multidimensional signals, such as those arising in image and video processing. In MDSDF, each actor produces and consumes data in units of $n$-dimensional cubes, where $n$ can be arbitrary, and can differ from actor to actor. The "synchrony" requirement in MDSDF constrains each production and consumption $n$-cube to be of fixed size $s_{1} \times s_{2} \times \ldots \times s_{n}$, where each $s_{i}$ is a constant. For example, an image processing actor that expands a $512 \times 512$-pixel image segment into a $1024 \times 1024$ segment would have the MDSDF representation illustrated in fig. 11 .

We say that a dataflow computation model is statically schedulable if a static schedule can always be constructed for a consistent specification in the model. For SDF, CSDF, and MDSDF, binary consistency and static schedulability both hold. The well-behaved dataflow (WBDF) model [24], proposed by Gao, Govindarajan, and Panangaden, is an example of a binary-consistency model that is not statically schedulable. The WBDF model permits the use of a limited set of datadependent control-flow constructs, and thus requires dynamic scheduling, in general. However the use of these constructs is restricted in such a way that that the inter-related properties of binary-consistency and efficient bounded memory verification are preserved, and the construction of efficient quasi-static schedules is facilitated. 
The boolean dataflow (BDF) model [25] is an example of a DSP dataflow model for which binary consistency does not hold. BDF introduces the concept of control inputs, which are actor inputs that affect the number of tokens produced and consumed at other input/output ports. In BDF, the values of control inputs are restricted to the set $\{T, F\}$. The number of tokens consumed by an actor from a non-control input edge, or produced onto an output edge is restricted to be constant, as in SDF, or a function of one or more data values consumed at control inputs. BDF attains greatly increased expressive power by allowing data-dependent production and consumption rates. In exchange, some of the intuitive simplicity and appeal of SDF is lost; static scheduling cannot always be employed; and the problems of bounded memory verification and deadlock detection become undecidable [26], which means that in general, they cannot be solved in finite time. However, heuristics have been developed for constructing efficient quasi-static schedules, and attempting to verify bounded memory requirements. These heuristics have been shown to work well in practice [26]. A natural extension of BDF, called integer-controlled dataflow, that allows control tokens to take on arbitrary integer values has been explored in [27].

\subsection{Optimized synthesis of DSP software from dataflow specifications}

In section 2.1, we reviewed several dataflow models for high-level, block diagram specification of DSP systems. Among these models, SDF and the closely related SSDF model are the most mature. In this this section we examine fundamental trade-offs and algorithms involved in the synthesis of DSP software from SDF and SSDF graphs. Except for the vectorization approaches discussed in section 2.2.4, the techniques discussed in this section apply equally well to both SDF and SSDF. For clarity, we present these techniques uniformly in the context of SDF.

\subsubsection{Threaded implementation of dataflow graphs}

A software synthesis tools generates application programs by piecing together code modules from a predefined library of software building blocks. These code modules are defined in terms of the target language of the synthesis tool. Most SDFbased design systems use a model of synthesis called threading. Given an SDF representation of a block-diagram program specification, a threaded synthesis tool begins by constructing a periodic schedule. The synthesis tool then steps through the schedule and for each actor instance $A$ that it encounters, it inserts the associated code module $A_{m}$ from the given library (inline threading), or inserts a call to a subroutine that invokes $A_{m}$ (subprogram threading). Threaded tools may employ purely inline threading, purely subroutine threading, or a mixture of inline and subprogram-based instantiation of actor functionality (hybrid threading). The sequence of code modules / subroutine calls that is generated from a dataflow graph is processed by a buffer management phase that inserts the necessary target program statements to route data appropriately between actors.

\subsubsection{Scheduling tradeoffs}

In this section, we provide a glimpse at the complex range of trade-offs that are involved during the scheduling phase of the synthesis process. At present, we consider only inline threading. Subprogram and hybrid threading are considered in section 2.2.5. Synthesis techniques that pertain to SSDF, which are discussed in section 2.2.4, can be applied with similar effectiveness to inline, subprogram or hybrid threading.

Scheduling is a critical task in the synthesis process. In a software implementation, scheduling has a large impact on key metrics such as program and data memory requirements, performance, and power consumption. Even for a simple SDF graph, the underlying range of trade-offs may be very complex. For example, consider the SDF graph in fig. 12(a). The repetitions vector components for this graph are $q(X)=1, q(Y)=q(Z)=10$. One possible schedule for this graph is given by

$$
S_{1}=Y Z Y Z Y Z Y Z Y Z X Y Z Y Z Y Z Y Z Y Z
$$


This schedule exploits the additional scheduling flexibility offered by the delays placed on edge $(X, Y)$. Recall that each delay results in an initial data value on the associated edge. Thus, in fig. 12, five executions of $Y$ can occur before $X$ is invoked, which leads to a reduction in the amount of memory required for data buffering.

To discuss such reductions in buffering requirements precisely, we need a few definitions. Given a schedule, the buffer size of an SDF edge is the maximum number of live tokens (tokens that are produced but not yet consumed) that coexist on the edge throughout execution of the schedule. The buffer requirement of a schedule $S$, denoted buf $(S)$, is the sum of the buffer sizes of all of the edges in the given SDF graph. For example, it is easily verified that $\operatorname{buf}\left(S_{1}\right)=11$.

The quantity $b u f(S)$ is the number of memory locations required to implement the dataflow buffers in the input SDF graph assuming that each buffer is mapped to a separate segment of memory. This is a natural and convenient model of buffer implementation. It is used in SDF design tools such as Cadence's SPW and the SDF-related code generation domains of Ptolemy, Furthermore, scheduling techniques that employ this buffering model do not preclude the sharing of memory locations across multiple, non-interfering edges (edges whose lifetimes do not overlap): the resulting schedules can be postprocessed by any general technique for array memory allocation, such as the well-known first-fit or best-fit algorithms. In this case, the scheduling techniques, which attempt to minimize the sum of the individual buffer sizes, employ a buffer memory metric that is an upper bound approximation to the final buffer memory cost.

One problem with the schedule $S_{1}$ under the assumed inline threading model is that it consumes a relatively large amount of program memory. If $\kappa(A)$ denotes the code size (number of program memory words required) for an actor $A$, then the code size cost of $S_{1}$ can be expressed as $\kappa(X)+10 \kappa(Y)+10 \kappa(Z)$.

By exploiting the repetitive subsequences in the schedule to organize compact looping structures, we can reduce the code size cost required for the actor execution sequence implemented by $S_{1}$. The structure of the resulting software implementation can be represented by the looped schedule

$$
S_{2}=(5 Y Z) X(5 Y Z)
$$

Each parenthesized term $\left(n T_{1} T_{2} \ldots T_{m}\right)$ (called a schedule loop) in such a looped schedule represents the successive repetition $n$ times of the invocation sequence $T_{1} T_{2} \ldots T_{m}$. Each iterand $T_{i}$ can be an instantiation (appearance) of an actor, or a looped subschedule. Thus, this notation naturally accommodates nested loops.

Given an arbitrary firing sequence $F$ (that is, a schedule that contains no schedule loops), and a set of code size costs for all of the given actors, a looped schedule can be derived that minimizes the total code size (over all looped schedules that have $F$ as the underlying firing sequence) using an efficient dynamic programming algorithm [28] called CDPPO. It is easily verified that the schedule $S_{2}$ achieves the minimum total code size for the firing sequence $S_{1}$ for any given values of $\kappa(X)$, $\kappa(Y)$, and $\kappa(Z)$. In general, however, the the set of looped schedules that minimize the code size cost for a firing sequence may depend on the relative costs of the individual actors [28].

Schedules $S_{1}$ and $S_{2}$ both attain the minimum achievable buffer requirement of 11 for fig. 12; however, $S_{2}$ will generally achieve a much lower code size cost. The code size cost of $S_{2}$ can be approximated as $\kappa(X)+2 \kappa(Y)+2 \kappa(Z)$. This approximation neglects the code size overhead $\lambda\left(S_{2}\right)$ of implementing the schedule loops (parenthesized terms) within $S_{2}$. In practice, this approximation rarely leads to misleading results. The looping overhead is typically very small compared to the code size saved by consolidating actor appearances in the schedule. This is especially true for the large number of DSP processors that employ so-called "zero-overhead looping" facilities [2]. Scheduling techniques that abandon this approximation, and incorporate looping overhead are examined in section 2.2.5.

It is possible to reduce the code size cost below what is achievable by $S_{1}$; however, this requires an increase in the buffering cost. For example, consider the schedule $S_{3}=X(10 Y)(10 Z)$. Such a schedule is called a single appearance schedule since it contains only one instantiation of each actor. Clearly (under the approximation of negligible looping overhead), any single appearance schedule gives a minimal code size implementation of a dataflow graph. However, a penalty in the buffer requirement must usually paid for such code size optimality. 
For example, the code size cost of $S_{3}$ is $(\kappa(X)+\kappa(Y))$ less than that of $S_{2}$; however buf $\left(S_{3}\right)=25$, while buf $\left(S_{2}\right)$ is only 11 .

Beyond code size optimality, another potentially important benefit of schedule $S_{3}$ is that it minimizes the average rate at which inter-actor context switching occurs. This schedule incurs 3 context switches (also called actor activations) per schedule period, while $S_{1}$ and $S_{2}$ both incur 21. Such minimization of context switching can significantly improve throughput and power consumption. The issue of context switching, and the systematic construction of minimum-context-switch schedules are discussed further in section 2.2.4.

An alternative single appearance schedule for fig. 12 is $S_{4}=X(10 Y Z)$. This schedule has the same optimal code size cost as $S_{3}$. However its buffer requirement of 16 is lower than that of $S_{3}$ since execution of actors $Y$ and $Z$ is fully interleaved, which limits data accumulation on the edge $(Y, Z)$. This interleaving, however, brings the average rate of context switches to 21; and thus, $S_{3}$ is clearly advantageous in terms of this metric.

In summary, there is a wide, complex range of trade-offs involved in synthesizing an application program from a dataflow specification. This is true even when we restrict ourselves to inline implementations, which entirely avoid the (call/return/parameter passing) overhead of subroutines. In the remainder of this section, we review a number of techniques that have been developed for addressing some of these complex trade-offs. Sections 2.2.3 and 2.2.4 focus primarily on inline implementations. In section 2.2.5, we examine some recently-developed techniques that have been developed to incorporate subroutine-based threading into the design space.

\subsubsection{Minimization of memory requirements}

Minimizing program and data memory requirements is critical in many embedded DSP applications. On-chip memory capacities are limited, and the speed, power, and financial cost penalties of employing off-chip memory may be prohibitive or highly undesirable. Three general avenues have been investigated for minimizing memory requirements - minimization of the buffer requirement, which usually forms a significant component of the over all data space cost; minimization of code size; and joint exploration of the trade-off involving code size and buffer requirements.

It has been shown that the problem of constructing a schedule that minimizes the buffer requirement over all valid schedules is NP-complete [11]. Thus, for practical, scalable algorithms, we must resort to heuristics. Ade [29] has developed techniques for computing tight lower bounds on the buffer requirement for a number of restricted subclasses of delayless, acyclic graphs, including arbitrary-length chain-structured graphs. Some of these bounds have been generalized to handle delays in [11]. Approximate lower bounds for general graphs are derived in [30]. Cubric and Panangaden have presented an algorithm that achieves optimum buffer requirements for acyclic SDF graphs that may have one or more independent, undirected cycles [31]. An effective heuristic for general graphs, which is employed in the Gabriel [14] and Ptolemy [7] systems, is given in [11]. Govindarajan, Gao, and Desai have developed an SDF buffer minimization algorithm for multiprocessor implementation [32]. This algorithm minimizes the buffer memory cost over all multiprocessor schedules that have optimal throughput.

For complex, multirate applications - which are the most challenging for memory management - the structure of minimum buffer schedules is in general highly irregular [33,11]. Such schedules offer relatively few opportunities to organize compact loop structures, and thus have very high code size costs under inlined implementations. Thus, such schedules are often not useful even though they may achieve very low buffer requirements. Schedules at the extreme of minimum code size, on the other hand, typically exhibit a much more favorable trade-off between code and buffer memory costs [34].

These empirical observations motivate the problem of code size minimization. A central goal when attempting to minimize code size for inlined implementations is that of constructing a single appearance schedule whenever one exists. A valid single appearance schedule exists for any consistent, acyclic SDF graph. Furthermore, a valid single appearance schedule can be derived easily from any topological sort (a topological sort of a directed acyclic graph $G$ is a linear ordering of all its vertices such that for each edge $(x, y)$ in $G, x$ appears before $y$ in the ordering) of an acyclic graph $G$ : if $\left(A_{1}, A_{2}, \ldots, A_{m}\right)$ 
is a topological sort of $G$, then it is easily seen that the single appearance schedule $\left(q\left(A_{1}\right) A_{1}\right)\left(q\left(A_{2}\right) A_{2}\right) \ldots\left(q\left(A_{m}\right) A_{m}\right)$ is valid. For a cyclic graph, a single appearance schedule may or may not exist depending on the location and magnitude of delays in the graph. An efficient strategy, called the Loose Interdependence Algorithm Framework (LIAF), has been developed that constructs a single appearance schedule whenever one exists [35]. Furthermore, for general graphs, this approach guarantees that all actors that are not contained in a certain type of subgraph, called tightly interdependent subgraphs, will have only one appearance in the generated schedule [36]. In practice, tightly interdependent subgraphs arise only very rarely, and thus, the LIAF technique guarantees full code size optimality for most applications. Because of its flexibility and provable performance, the LIAF is employed in a number of widely used tools, including Ptolemy and Cadence's SPW.

The LIAF constructs a single appearance schedule by decomposing the input graph into a hierarchy of acyclic subgraphs, which correspond to an outer-level hierarchy of nested loops in the generated schedule. The acyclic subgraphs in the hierarchy can be scheduled with any existing algorithm that constructs single appearance schedules for acyclic graphs. The particular algorithm that is used in a given implementation of the LIAF is called the acyclic scheduling algorithm. For example, the topological-sort-based approach described above could be used as the acyclic scheduling algorithm. However, this simple approach has been shown to lead to relatively large buffer requirements [11]. This motivates a key problem in the joint minimization of code and data for SDF specifications. This is the problem of constructing a single appearance schedule for an acyclic SDF graph that minimizes the buffer requirement over all valid single appearance schedules. Since any topological sort leads to a distinct schedule for an acyclic graph, and the number of topological sorts is not polynomially bounded in the graph size, exhaustive evaluation of single appearance schedules is not tractable. Thus, as with the (arbitrary appearance) buffer minimization problem, heuristics have been explored. Two complementary, low-complexity heuristics, called APGAN [37] and RPMC [38], have proven to be effective on practical applications when both are applied, and the best resulting schedule is selected. Furthermore, it has been formally shown that APGAN gives optimal results for a broad class of SDF systems. Thorough descriptions of APGAN, RPMC, and the LIAF, and their inter-relationships can be found in [11,34]. A scheduling framework for applying these techniques to multiprocessor implementations is described in [39]. Recently-developed techniques for efficient sharing of memory among multiple buffers from a single appearance schedule are developed in [40, 41].

Although APGAN and RPMC provide good performance on many applications, these heuristics can sometimes produce results that are far from optimal [42]. Furthermore, as discussed in section 1, DSP software tools are allowed to spend more time for optimization of code than what is required by low-complexity, deterministic algorithms such as APGAN and RPMC. Motivated by these observations, Zitzler, Teich, and Bhattacharyya have developed an effective stochastic optimization methodology, called GASAS, for constructing minimum buffer single appearance schedules [43, 44]. The GASAS approach is based on a genetic algorithm [45] formulation in which topological sorts are encoded as "chromosomes," which randomly "mutate" and "recombine" to explore the search space. Each topological sort in the evolution is optimized by the efficient, local search algorithm CDPPO [28], which was mentioned earlier in section 2.2.2. Using dynamic programming, CDPPO computes a minimum memory single appearance schedule for a given topological sort. To exploit the valuable optimality property of APGAN whenever it applies, the solution generated by APGAN is included in the initial population, and an elitist evolution policy is enforced to ensure that the fittest individual always survives to the next generation.

\subsubsection{Throughput optimization}

At the Aachen University of Technology, as part of the COSSAP design environment (now developed by Synopsys) project, Ritz, Pankert, and Meyr have investigated the minimization of of the context-switch overhead, or the average rate at which actor activations occur [20]. As discussed in section 2.2.2, an actor activation occurs whenever two distinct actors are invoked in succession; for example, the schedule $(2(2 B)(5 A))(5 C)$ for fig. 13 results in five activations per schedule period.

Activation overhead includes saving the contents of registers that are used by the next actor to invoke, if necessary, and loading state variables and buffer pointers into registers. The concept of grouping multiple invocations of the same actor 
together to reduce context-switch overhead is referred to as vectorization. The SSDF model, discussed in section 2.1.6, allows the benefits of vectorization to extend beyond the actor interface level (inter-actor context switching). For example, context switching between successive sub-functions of a complex actor can be amortized over $N_{v}$ invocations of the sub-functions, where $N_{v}$ is the given vectorization parameter.

Ritz estimates the average rate of activations for a periodic schedule $S$ as the number of activations that occur in one iteration of $S$ divided by the blocking factor ${ }^{1}$ of $S$. This quantity is denoted by $N_{\text {act }}(S)$ For example, for fig. 13, $N_{a c t}((2(2 B)(5 A))(5 C))=5$, and $N_{a c t}((4(2 B)(5 A))(10 C))=9 / 2=4.5$. If for each actor, each invocation takes the same amount of time, and if we ignore the time spent on computation that is not directly associated with actor invocations (for example, schedule loops), then $N_{a c t}(S)$ is directly proportional to the number of actor activations per unit time. For consistent acyclic SDF graphs, $N_{a c t}$ clearly can be made arbitrarily large by increasing the blocking factor sufficiently; thus, as with the problem of constructing compact schedules, the extent to which the activation rate can be minimized is limited by the cyclic regions in the input SDF specification.

The technique developed in [20] attempts to find a valid single appearance schedule that minimizes $N_{a c t}$ over all valid single appearance schedules. Note that minimizing the number of activations does not imply minimizing the number of appearances. As a simple example, consider the SDF graph in fig. 14. It can be verified that for this graph, the lowest value of $N_{a c t}$ that is obtainable by a valid single appearance schedule is 0.75 , and one valid single appearance schedule that achieves this minimum rate is $(4 B)(4 A)(4 C)$. However, valid schedules exist that are not single appearance schedules, and that have values of $N_{\text {act }}$ below 0.75; for example, the valid schedule $(4 B)(4 A)(3 B)(3 A)(7 C)$ contains two appearances each of $A$ and $B$, and satisfies $N_{a c t}=5 / 7=0.71$.

Thus, since Ritz's vectorization approach focuses on single appearance schedules, the primary objective of the techniques in [20] is implicitly code size minimization. This is reasonable since in practice, code size is often of critical concern. The overall objective is in [20] is to construct a minimum activation implementation over all implementations that have minimum code size.

Ritz defines the relative vectorization degree of a simple cycle (a cyclic path in the graph in which no proper sub-path is cyclic) $C$ in a consistent, connected SDF graph by

$$
\begin{array}{r}
N_{G}(C)=\max \left(\left\{\min \left(\left\{D_{G}(\beta) \mid \beta \in \operatorname{parallel}(\alpha)\right\}\right) \mid\right.\right. \\
\alpha \in \operatorname{edges}(C)\}),
\end{array}
$$

where

$$
D_{G}(\alpha)=\left\lfloor\frac{\operatorname{del}(\alpha)}{q(\operatorname{src}(\alpha)) \operatorname{prd}(\alpha)}\right\rfloor
$$

is the delay on edge $\alpha$ normalized by the total number of tokens exchanged on $\alpha$ in a minimal schedule period of $G$, and

$$
\begin{gathered}
\operatorname{parallel}(\alpha)=\{\beta \in \text { edges }(G) \mid \\
(\operatorname{src}(\beta)=\operatorname{src}(\alpha)) \text { and }(\operatorname{snk}(\beta)=\operatorname{snk}(\alpha))\}
\end{gathered}
$$

is the set of edges with the same source and sink as $\alpha$. Here, edges $(G)$ simply denotes the set of edges in the SDF graph $G$.

For example, if $G$ denotes the SDF graph in fig. 13, and $\chi$ denotes the cycle in $G$ whose associated graph contains the actors $A$ and $B$, then $D_{G}(\chi)=\lfloor 10 / 20\rfloor=0$; and if $G$ denotes the graph in fig. 14 and $\chi$ denotes the cycle whose associated graph contains $A$ and $C$, then $D_{G}(\chi)=\lfloor 7 / 1\rfloor=7$.

\footnotetext{
${ }^{1}$ Every periodic schedule invokes each actor $A$ some multiple of $q(A)$ times. This multiple, denoted by $J$, is called the blocking factor. A minimal periodic schedule is one that satisfies $J=1$. For memory minimization, there is no penalty in restricting consideration to minimal schedules [11]. When attempting to minimize $N_{a c t}$, however, it is in general advantageous to consider $J>1$.
} 
Ritz et. al postulate that given a strongly connected SDF graph, a valid single appearance schedule that minimizes $N_{a c t}$ can be constructed from a complete hierarchization, which is a cluster hierarchy such that only connected subgraphs are clustered, all cycles at a given level of the hierarchy have the same relative vectorization degree, and cycles in higher levels of the hierarchy have strictly higher relative vectorization degrees than cycles in lower levels. Fig. 15 depicts a complete hierarchization of an SDF graph. Fig. 15(a) shows the original SDF graph; here $q(A, B, C, D)=(1,2,4,8)$. Fig. 15(b) shows the top level of the cluster hierarchy. The hierarchical actor $\Omega_{1}$ represents $\operatorname{subgraph}(\{B, C, D\})$, and this subgraph is decomposed as shown in fig. 15(c), which gives the next level of the cluster hierarchy. Finally, fig. 15(d) shows that $\operatorname{subgraph}(\{C, D\})$ corresponds to $\Omega_{2}$ and is the bottom level of the cluster hierarchy.

Now observe that the relative vectorization degree of the fundamental cycle in fig. 15(c) with respect to the original SDF graph is $\lfloor 16 / 8\rfloor=2$, while the relative vectorization degree of the fundamental cycle in fig. 15 (b) is $\lfloor 12 / 2\rfloor=6$; and the relative vectorization degree of the fundamental cycle in fig. 15 (c) is $\lfloor 12 / 8\rfloor=1$. We see that the relative vectorization degree decreases as we descend the hierarchy, and thus the hierarchization depicted in fig. 15 is complete. The hierarchization step defined by each of the SDF graphs in figs. 15(b)-(d) is called a component of the overall hierarchization.

Ritz's algorithm [20] constructs a complete hierarchization by first evaluating the relative vectorization degree of each fundamental cycle, determining the maximum vectorization degree, and then clustering the graphs associated with the fundamental cycles that do not achieve the maximum vectorization degree. This process is then repeated recursively on each of the clusters until no new clusters are produced. In general, this bottom-up construction process has unmanageable complexity. However, this normally doesn't create problems in practice since the strongly connected components of useful signal processing systems are often small, particularly in large grain descriptions. Details on Ritz's technique for translating a complete hierarchization into a hierarchy of nested loops can be found in [20]. A general, optimal algorithm for vectorization of SSDF graphs based on the complete hierarchization concept discussed above is given in [20]. Joint minimization of vectorization and buffer memory cost is developed in [12], and adaptations of the retiming transformation to improve vectorization for SDF graphs is addressed in $[46,47]$.

\subsubsection{Subroutine insertion}

The techniques discussed above assume a fixed threading mode. In particular, they do not attempt to exploit the flexibility offered by hybrid threading. Sung, Kim, and Ha have developed an approach that employs hybrid threading to share code among different actors that have similar functionality [48]. For example, an application may contain several FIR filter blocks that differ only in the number of taps, and the set of filter coefficients. These are called different instances of a parameterized FIR module in the actor library. Their approach decomposes the code associated with an actor instance into the actor context and actor reference code, and carefully weighs the benefit of each code sharing opportunity with the associated overhead. The overheads stem from the actor context component, which include instance-specific state variables, and buffer pointers. Code must be inserted to manage this context so that each invocation of the shared code block (the "reference code") is appropriately customized to the associated instance.

Also, the GASAS framework has been significantly extended to consider multiple appearance schedules, and selectively apply hybrid threading to reduce the code size cost of highly irregular schedules, which cannot be accommodated by compact loop structures [49]. Such irregularity often arises when exploring the space of schedules whose buffer requirements are significantly lower than what is achievable by single appearance schedules [11]. The objective of this genetic-algorithmbased exploration of hybrid threading and loop scheduling is to efficiently compute Pareto-fronts in the multidimensional design evaluation space of program memory cost, buffer requirement, and execution time overhead.

The intelligent use of hybrid threading and code sharing (subroutine insertion optimizations) can achieve lower code size costs that what is achievable with single appearance schedules that use conventional inlining. If an inlined single appearance schedule fits within the available on-chip memory, it is not worth incurring the overhead of subroutine insertion. However, if an inline implementation is too large to be held on-chip, then subroutine insertion optimizations can eliminate, or greatly 
reduce the need for off-chip memory accesses. Since off-chip memory accesses involve significant execution time penalties, and large power consumption costs, subroutine insertion enables embedded software developers to exploit an important part of the design space.

\subsubsection{Summary}

In this section we have reviewed a variety of algorithms for addressing optimization trade-offs during software synthesis. We have illustrated some of the analytical machinery used in SDF optimization algorithms by examining in some detail Ritz's algorithm for minimizing actor activations. Since CSDF, MDSDF, WBDF, and BDF are extensions of SDF, the techniques discussed in this section can also be applied in these more general models. In particular, they can be applied to any SDF subgraphs that are found. It is important to recognize this when developing or using a DSP design tool since in DSP applications that are not fully amenable to SDF semantics, a significant subset of the functionality can usually be expressed in SDF. Thus the techniques discussed in this section remain useful even in DSP tools that employ more general dataflow semantics.

Beyond their application to SDF subsystems, however, the extension of most of the techniques developed in this section to more general dataflow models is a non-trivial matter. To achieve best results with these more general models, new synthesis approaches are required that take into account distinguishing characteristics of the models. The most successful approaches will combine these new approaches for handling the full generality of the associated models, with the techniques that exploit the structure of pure SDF subsystems.

\section{Compilation of application programs to machine code}

In this section, we will first outline the state of the art in the area of compilers for PDSPs. As indicated by several empirical studies, the major problem with current compiler is their inability to generate machine code of sufficient quality. Next, we will discuss a number of recently developed code generation and optimization techniques, which explicitly take into account DSPspecific architectures and requirements in order to improve code quality. Finally, we will mention key techniques developed for retargetable compilation.

\subsection{State of the art}

Today, the most widespread high-level programming language for PDSPs is ANSI C. Even though there are more DSPspecific languages, such as the data flow language DFL [50], the popularity and high flexibility of $\mathrm{C}$ as well as the large amount of existing "legacy code" has so far largely prevented the use of programming languages more suitable for DSP programming. C compilers are available for all important DSP families, such as Texas Instruments TMS320xx, Motorola 56xxx, or Analog Devices 21xx. In most cases, the compilers are provided by the semiconductor vendors themselves.

Due to the large semantical gap between the $\mathrm{C}$ language and PDSP instruction sets, many of these compilers make extensions to the ANSI C standard by permitting the use of "compiler intrinsics", for instance in the form of compiler-known functions which are expanded like macros into specific assembly instructions. Intrinsics are used to manually guide the compiler in making the right decisions for generation of efficient code. However, such an ad-hoc approach has significant drawbacks. First, the source code deviates from the language standard and is no longer machine-independent. Thus, porting of software to another processor might be a very time-consuming task. Second, the programming abstraction level is lowered and the efficient use of compiler intrinsics requires a deep knowledge of the internal PDSP architecture.

Unfortunately, machine-specific source code today is a must whenever the $\mathrm{C}$ language is used for programming PDSPs. The reason is the poor quality of code generated by compilers from plain ANSI C code. The overhead of compiler-generated code as compared to hand-written, heavily optimized assembly code has been quantified in the DSPStone benchmarking project [6]. In that project, both code size and performance of compiler-generated code have been evaluated for a number 
of DSP kernel routines and different PDSP architectures. The results showed that the compiler overhead typically ranges between 100 and $700 \%$ (with the reference assembly code set to $0 \%$ overhead). This is absolutely insufficient in the area of DSP, where real-time constraints as well as limitations on program memory size and power consumption demand for an extremely high utilization of processor resources. Therefore, an overhead of compiler-generated code close or equal to zero is most desirable.

In another empirical study [51], DSP vendors have been asked to compile a set of $\mathrm{C}$ benchmark programs existing in two different versions, one being machine-independent and the other being tuned for the specific processor. Again, the results showed that using machine-independent code causes an unacceptable overhead in code quality in terms of code size and performance.

These results make the practical use of compilers for PDSP software development questionable. In the area of general purpose processors, such as RISCs, the compiler overhead typically does not exceed $100 \%$, so that even for DSP applications using a RISC together with a good compiler may result in a more efficient implementation than using a PDSP (with potentially much higher performance) wasting most of its time executing unnecessary instruction cycles due to a poor compiler. Similar arguments hold, if code size or power consumption are of major concern.

As a consequence, the largest part of PDSP software is still written in assembly languages, which implies a lot of wellknown drawbacks, such as high development costs, low portability, and high maintenance and debugging effort. This has been quantified in a study by Paulin [52], who found that for a certain set of DSP applications about $90 \%$ of DSP code lines are written in assembly, while the use of $\mathrm{C}$ only accounts for $10 \%$.

As both DSP processors and DSP applications tend to become more and more complex, the lack of good C compilers implies a significant productivity bottleneck. About a decade ago, researchers started to analyze the reasons for the poor code quality of DSP compilers. A key observation was that classical code generation technology, mainly developed for RISC and CISC processor architectures, is hardly suitable for PDSPs, but that new DSP-specific code generation techniques were required. In the following, we will summarize a number of recent techniques. In order to put these techniques into context with each other, we will first give an overview about the main phases in compilation. Then, we will focus on techniques developed for particular problems in the different compilation phases.

\subsection{Overview of the compilation process}

The compilation of an application program into machine code, as illustrated in fig. 16, starts with several source code analysis phases.

Lexical analysis: The character strings denoting atomic elements of the source code (identifiers, keywords, operators, constants) are grouped into tokens, i.e. numerical identifiers, which are passed to the syntax analyzer. Lexical analysis is typically performed by a scanner, which is invoked by the syntax analyzer whenever a new token is required. Scanners can be automatically generated from a language specification with tools like "lex".

Syntax analysis: The structure of programming languages is mostly described by a context-free grammar, consisting of terminals (or tokens), nonterminals, and rules. The syntax analyzer, or parser, accepts tokens from the scanner, until a matching grammar rule is detected. Each rule corresponds to a primitive element of the programming language, for instance an assignment. If a token sequence does not match any rule, a syntax error is emitted. The result of parsing a program is a syntax tree, which accounts for the structure of a given program. Parsers can be conveniently generated from grammar specifications with tools like "yacc'.

Semantical analysis: During semantical analysis, a number of correctness tests are performed. For instance, all used identifiers must have been declared, and functions must be called with parameters in accordance with their interface specification. Failure of semantical analysis results in error messages. Additionally, a symbol table is built, which annotates 
each identifier with its type and purpose (e.g. type definition, global or local variable). Semantical analysis requires a traversal of the syntax tree. Frequently, semantical analysis is coupled with syntax analysis by means of attribute grammars. These grammars support the annotation of information like type or purpose to grammar symbols, and thus help to improve the modularity of analysis. Tools like "ox" [53] are available for automatic generation of combined syntax and semantical analyzers from grammar specifications.

The result of source code analysis is an intermediate representation (IR), which forms the basis for subsequent compilation phases. Both graph-based and statement-based IRs are in use. Graph-based IRs directly model the interdependencies between program operations, while statement-based IRs essentially consist of an assembly-like sequence of simple assignments (three-address code) and jumps.

In the next phase, several machine-independent optimizations are applied to the generated IR. A number of such IR optimizations have been developed in the area of compiler construction [54]. Important techniques include constant folding, common subexpression elimination, and loop-invariant code motion.

The techniques mentioned so far are largely machine-independent and may be used in any high-level language compiler. DSP-specific information comes into play only during the code generation phase, when the optimized IR is mapped to concrete machine instructions. Due to the specialized instruction sets of PDSPs, this is the most important phase with respect to code quality. Due to computational complexity reasons, code generation is in turn subdivided into different phases. It is important to note that for PDSPs this phase structuring significantly differs from compilers for general purpose processors. For the latter, code generation is traditionally subdivided into the following phases.

Code selection: The selection of a minimum set of instructions for a given IR with respect to a cost metric like performance (execution cycles) or size (instruction words).

Register allocation: The mapping of variables and intermediate results to a limited set of available physical registers.

Instruction scheduling: The ordering of selected instructions in time while minimizing the number of instructions required for temporarily moving register contents to memory (spill code) and minimizing execution delay due to instruction pipeline hazards.

Such a phase organization is not viable for PDSPs due to several reasons. While general purpose processors often have a large, homogeneous register file, PDSPs tend to show a data path architecture with several distributed registers or register files of very limited capacity. An example has already been given in fig. 1. Therefore, classical register allocation techniques like [55] are not applicable, but register allocation has to be performed together with code selection in order to avoid large code quality overheads due to superfluous data moves between registers. Furthermore, instruction scheduling for PDSPs has to take into account the moderate degree of instruction-level parallelism (ILP) offered by such processors. In many cases, several mutually independent instructions may be grouped to be executed in parallel, thereby significantly increasing performance. This parallelization of instructions is frequently called code compaction. Another important area of code optimization for PDSPs concerns the memory accesses performed by a program. Both the exploitation of potentially available multiple memory banks and the efficient computation of memory addresses under certain restrictions imposed by the processor architecture have to be considered, which are hardly issues for general purpose processors. We will therefore discuss techniques using a different structure of code generation phases.

Sequential code generation: Even though PDSPs generally permit the execution of multiple instructions in parallel, it is often reasonable to temporarily consider a PDSP as a sequential machine, which executes instructions one-by-one. During sequential code generation, IR blocks (statement sequences) are mapped to sequential assembly code. These blocks are typically basic blocks, where control flow enters the block at its beginning and leaves the block at most once at its end with a jump. Sequential code generation aims at simultaneously minimizing the costs of instructions both for operations and data moves between registers and memory while neglecting ILP. 
Memory access optimization: Generation of sequential code makes the order of memory accesses in a program known. This knowledge is exploited to optimize memory access bandwidth by partitioning the variables among multiple memory banks and to minimize the additional code needed for address computations.

Code compaction: This phase analyzes interdependencies between generated instructions and aims at exploiting potential parallelism between instructions under the resource constraints imposed by the processor architecture and the instruction format.

\subsection{Sequential code generation}

Basic blocks in the IR of a program are graphically represented by data flow graphs (DFGs). A DFG $G=(V, E)$ is a directed acyclic graph, where the nodes in $V$ represent operations (arithmetic, Boolean, shifts, etc.), memory accesses (loads and stores), and constants. The edge set $E \subseteq V \times V$ represents the data dependencies between DFG nodes. If an operation represented by a node $w$ requires a value generated by an operation denoted by $v$, then $(v, w) \in E$. DFG nodes with more than one outgoing edge are called common subexpressions (CSEs). As an example, fig. 17 shows a piece of $\mathrm{C}$ source code, whose DFG representation (after detection of CSEs) is depicted in fig. 18.

Code generation for DFGs can be visualized as a process of covering a DFG by available instruction patterns. Let us consider a processor with instructions ADD, SUB, and MUL, to perform addition, subtraction, and multiplication, respectively. One of the operands is expected to reside in memory, while the other one has to be first loaded into a register by a LOAD instruction. Furthermore, writing back a result to memory requires a separate STORE instruction. Then, a valid covering of the example DFG is then one shown in fig. 19.

Available instruction patterns are usually annotated with a cost value reflecting their size or execution speed. The goal of code generation is to find a minimum cost covering of a given DFG by instruction patterns. The problem is that in general there exist numerous different alternative covers for a DFG. For instance, if the processor offers a MAC (multiply-accumulate) instruction, as found in most PDSPs, and the cost value of MAC is less than the sum of the costs of MUL and ADD, then it might be favorable to select that instruction (fig. 20).

However, using MAC for our example DFG would be less useful, because the multiply operation in this case is a CSE. Since the intermediate multiply result of a MAC is not stored anywhere, a potentially costly recomputation would be necessary.

\subsubsection{Tree based code generation}

Optimal code generation for DFGs is an exponential problem, even for very simple instruction sets [54]. A solution to this problem is to decompose a DFG into a set of data flow trees (DFTs) by cutting the DFG at its CSEs and inserting dedicated DFG nodes for communicating CSEs between the DFTs (fig. 21). This decomposition introduces scheduling precedences between the DFTs, since CSEs must be written before they are read (dashed arrows in fig. 21). For each of the DFTs, code can be generated separately and efficiently. Liem [57] has proposed a data structure for efficient tree pattern matching capable of handling complex operations like MAC.

For PDSPs, also the allocation of special purpose registers during DFT covering is extremely important, since only covering the operators in a DFG by instruction patterns does not take into account the costs of instructions needed to move operands and results to their required locations. Wess [58] has proposed the use of trellis diagrams to also include data move costs during DFT covering.

Araujo and Malik [60] showed how the powerful standard technique of tree pattern matching with dynamic programming [56] widely used in compilers for general purpose processors can be effectively applied also to PDSPs with irregular data paths. Tree pattern matching with dynamic programming solves the code generation problem by parsing a given DFT with respect to an instruction-set specification given as a tree grammar. Each rule in such a tree grammar is attributed with a cost 
value and corresponds to one instruction pattern. Optimal DFT covers are obtained by computing an optimal derivation of a given DFT according to the grammar rules. This requires only two passes (bottom-up and top-down) over the nodes of the input DFT, so that the runtime is linear in the number of DFT nodes. Code generators based on this paradigm can be automatically generated with tools like "twig" [56] and "iburg" [59].

The key idea in the approach by Araujo and Malik is the use of register-specific instruction patterns or grammar rules. Instead of separating detailed register allocation from code selection as in classical compiler construction, the instruction patterns contain implicit information on the mapping of operands and results to special purpose registers. In order to illustrate this, we consider an instruction subset of the TI TMS320C25 DSP already mentioned in section 1 (see also fig. 1. This PDSP offers two types of instructions for addition. The first one (ADD) adds a memory value to the accumulator register ACCU, while the second one (APAC) adds the value of the product register PR to ACCU. In compilers for general purpose processors, a distinction of storage components is made only between (general purpose) registers and memory. In a grammar model used for tree pattern matching with dynamic programming, the above two instructions would thus be modeled as follows:

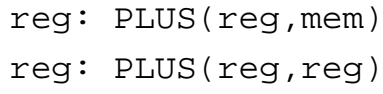

The symbols "reg" and "mem" are grammar nonterminals, while "PLUS" is a grammar terminal symbol representing an addition. The semantics of such rules is that the corresponding instruction computes the expression on the right hand side and stores the result in a storage component represented by the left hand side. When parsing a DFT with respect to these patterns it would be impossible to incorporate the costs of moving values to/from ACCU and PR, but the detailed mapping of "reg" to physical registers would be left to a later code generation phase, possibly at the expense of code quality losses. However, when using register-specific patterns, instructions ADD and APAC would be modeled as:

accu: PLUS (accu, mem)

accu: PLUS (accu, pr)

Using a separate nonterminal for each special purpose register permits to model instructions for pure data moves, which in turn allows the code generator to simultaneously minimize the costs of such instructions. As an example, consider the TMS320C25 instruction PAC, which moves a value from PR to ACCU. In the tree grammar, the following rule (a so-called chain rule) for PAC would be included:

accu: pr

Since using the PAC rule for derivation of a DFT would incur additional costs, the code generator implicitly minimizes the data moves when constructing the optimal DFT derivation.

Generation of sequential assembly code also requires to determine a total ordering of selected instructions in time. DFGs and DFTs typically only impose a partial ordering, and the remaining scheduling freedom must be exploited carefully. This is due to the fact, that special purpose registers generally have very limited storage capacity. On the TMS320C 25 , for instance, each register may hold only a single value, so that unfavorable scheduling decisions may require to spill and reload register contents to/from memory, thereby introducing additional code. In order to illustrate the problem, consider a DFT $T$ whose root node represents an addition, for which the above APAC instruction has been selected. Thus, the addition operands must reside in registers ACCU and PR, so that the left and right subtrees $T_{1}$ and $T_{2}$ of $T$ must deliver their results in these registers. When generating sequential code for $T$, it must be decided whether $T_{1}$ or $T_{2}$ should be evaluated first. If some instruction in $T_{1}$ writes its result to PR, then $T_{1}$ should be evaluated first in order to avoid a spill instruction, because $T_{2}$ writes its result to PR as well and this value is "live" until the APAC instruction for the root of $T$ is emitted. Conversely, if some instruction for $T_{2}$ writes register ACCU, then $T_{2}$ should be scheduled first in order to avoid a register contention for ACCU. In [60], Araujo and Malik formalized this observation and provided a formal criterion for the existence of a spill-free schedule for a given DFT. This criterion refers to the structure of the instruction set and, for instance, holds for the TMS320C25. When using an 
appropriate scheduling algorithm, which immediately follows from that criterion, then optimal spill-free sequential assembly code can be generated for any DFT.

\subsubsection{Graph based code generation}

Unfortunately, the DFT-based approach to code generation may affect code quality, because it performs only a local optimization of code for a DFG within the scope of the single DFTs. Therefore, researchers have investigated techniques aiming at optimal or near-optimal code generation for full DFGs. Liao [61] has presented a branch-and-bound algorithm minimizing the number of spills in accumulator-based machines, i.e. processors where most computed values have to pass a dedicated accumulator register. In addition, his algorithm minimizes the number of instructions needed for switching between different computation modes. These modes (e.g. sign extension or product shift modes) are special control codes stored in dedicated mode registers in order to reduce the instruction word length. If the operations within a DFG have to be executed with different modes, the sequential schedule has a strong impact on the number of instructions for mode switching. Liao's algorithm simultaneously minimizes accumulator spills and mode switching instructions. However, due to the time-intensive optimization algorithm, optimality cannot be achieved for large basic blocks. The code generation technique in [62] additionally performs code selection for DFGs, but also requires high compilation times for large blocks.

A faster heuristic approach has been given in [63]. It also relies on the decomposition of DFGs into DFTs, but takes into account architectural information when cutting the CSEs in a DFG. In some cases, the machine instruction set itself enforces that CSEs have to pass the memory anyway, which again is a consequence of the irregular data paths of PDSPs. The proposed technique exploits this observation by assigning those CSEs to memory with highest priority, while others might be kept in a register, resulting in more efficient code.

Kolson et al. [64] have focused on the problem of code generation for irregular data paths in the context of program loops. While the above techniques deal well with special purpose registers in basic blocks, the do not take into account the data moves required between different iterations of a loop body. This may require the execution of a number of data moves between those registers holding the results at the end of one iteration and those registers where operands are expected at the beginning of the next iteration. Both an optimal and a heuristic algorithm have been proposed for minimizing the data moves between loop iterations.

\subsection{Memory access optimization}

During sequential code generation, memory accesses are usually treated only "symbolically" without particular reference to a certain memory bank or memory addresses. The detailed implementation of memory accesses is typically left to a separate code generation phase.

\subsubsection{Memory bank partitioning}

There exist several PDSP families having the memory organized in two different banks (typically called X and Y memory), which are accessible in parallel. Examples are Motorola 56xxx and Analog Devices 21xx. Such an architecture allows to simultaneously load two values from memory into registers and is therefore very important for DSP applications like digital filtering or FFT, involving component-wise access to different data arrays. Exploiting this feature in a compiler means, that symbolic memory accesses have to be partitioned into $\mathrm{X}$ and $\mathrm{Y}$ memory accesses in such a way, that potential parallelism is maximized. Sudarsanam [65] has proposed a technique to perform this optimization. There is a strong mutual dependence between memory bank partitioning and register allocation, because values from a certain memory bank can only be loaded into certain registers. The proposed technique starts from symbolic sequential assembly code and uses a constraint graph model to represent these interdependencies. Memory bank partitioning and register allocation are performed simultaneously 
by labeling the constraint graph with valid assignments. Due to the use of simulated annealing, the optimization is rather time-intensive, but may result in significant code size improvements, as indicated by experimental data.

\subsubsection{Memory layout optimization}

As one cost metric, Sudarsanam's technique also captures the cost of instructions needed for address computations. For PDSPs which typically show very restricted address generation capabilities, address computations are another important area of code optimization. Fig. 22 shows the architecture of an address generation unit (AGU) as it is frequently found in PDSPs.

Such an AGU operates in parallel to the central data path and contains a separate adder/subtractor for performing operations on address registers (ARs). ARs store the effective addresses for all indirect memory accesses, except for global variables typically addressed in direct mode. Modify registers (MRs) are used to store frequently required address modify values. ARs and MRs are in turn addressed by AR and MR pointers. Since typical AR or MR file sizes are 4 or 8 , these pointers are short indices of 2 or 3 bits, either stored in the instruction word itself or in special small registers.

There are different means for address computation, i.e., for changing the value of AGU registers.

AR load: Loading an AR with an immediate constant (from the instruction word).

MR load: Loading a MR with an immediate constant.

AR modify: Adding or subtracting an immediate constant to/from an AR.

Auto-increment and auto-decrement: Adding or subtracting the constant 1 to/from an AR.

Auto-modify: Adding or subtracting the contents of one MR to/from an AR.

While details like the size of AR and MR files or the signed-ness of modify values may vary for different processors, the general AGU architecture from fig. 22 is actually found in a large number of PDSPs. It is important to note that performing address computations using the AGU in parallel to other instructions is generally only possible, if the AGU does not use the instruction word as a resource. The wide immediate operand for AR and MR load and AR modify operations usually leaves no space to encode further instructions within the same instruction word, so that these two types of AGU operations require a separate non-parallel instruction. On the other hand, those AGU operations not using the instruction word can mostly be executed in parallel to other instructions, since only internal AGU resources are occupied. We call these address computations zero-cost operations. In order to maximize code quality in terms of performance and size it is obviously necessary to maximize the utilization of zero-cost operations.

A number of techniques have been developed which solve this problem for the scalar variables in a program. They exploit the fact, that when the sequence of variable accesses is known after sequential code generation, a good memory layout for the variables can still be determined. In order to illustrate this, suppose a program block containing accesses to the variables

$$
V=\{a, b, c, d\}
$$

is given, and the variable access sequence is

$$
S=(b, d, a, c, d, a, c, b, a, d, a, c, d)
$$

Furthermore, let the address space reserved for $V$ be $A=\{0,1,2,3\}$ and let one AR be available to compute the addresses according to the sequence $S$. Consider a memory layout where $V$ is mapped to $A$ in lexicographic order (fig. 23 a).

First, AR needs to be loaded with the address 1 of the first element $b$ of $S$. The next access takes place to $d$ which is mapped to address 3. Therefore, AR must be modified with a value of +2 . The next access refers to $a$, which requires to subtract 3 from AR, and so forth. The complete AGU operation sequence for $S$ is given in fig. 23 a). According to our cost 
metric, only 4 out of $13 \mathrm{AGU}$ operations happen to be zero-cost operations (auto-increment or decrement), so that a cost of 9 extra instructions for address computations is incurred. However, one can find a better memory layout for $V$ (fig. 23 b), which leads to only 5 extra instructions, due to a better utilization of zero-cost operations. An even better addressing scheme is possible if a modify register MR is available. Since the address modifier 2 is required three times in the AGU operation sequence from fig. $23 \mathrm{~b}$ ), one can assign the value 2 to MR (one extra instruction) but reuse this value three times at zero cost (fig. 23 c), resulting in a total cost value of only 3.

How can such "low cost" memory layouts be constructed? A first approach has been proposed by Bartley [66] and has later been refined by Liao [67]. Both use an access graph to model the problem.

The nodes of the edge-weighted access graph $G=(V, E, w)$ correspond to the variable set, while the edges represent transitions between variable pairs in the access sequence $S$. An edge $e=(v, w) \in E$ is assigned an integer weight $n$, if there are $n$ transitions $(v, w)$ or $(w, v)$ in $S$. Fig. 24 shows the access graph for our example. Since any memory layout for $V$ implies a linear order of $V$ and vice versa, any memory layout corresponds to a Hamiltonian path in $G$, i.e., a path touching each node exactly once. Informally, a "good' Hamiltonian path obviously should contain as many edges of high weight as possible, because including these edges in the path implies that the corresponding variable pairs will be adjacent in the memory layout, which in turn makes auto-increment/decrement addressing possible. In other words, a maximum Hamiltonian path in $G$ has to be found, in order to obtain an optimal memory layout, which unfortunately is an exponential problem.

While Bartley [66] first proposed the access graph model, Liao [67] provided an efficient heuristic algorithm to find maximum paths in the access graph. Furthermore, Liao proposed a generalization of the algorithm for the case of an arbitrary number $k$ of ARs. By partitioning the variable set $V$ into $k$ groups, the $k$-AR problem is reduced to $k$ different 1-AR problems, each being solvable by the original algorithm.

Triggered by this work, a number of improvements an generalizations have been found. Leupers [68] improved the heuristic for the 1-AR case and proposed a more effective partitioning for the $k$-AR problem. Furthermore, he provided a first algorithm for the exploitation of MRs to reduce addressing costs. Wess' algorithm [69] constructs memory layouts for AGUs with an auto-increment range of 2 instead of 1, while in [70] a generalization for an arbitrary integer auto-increment range was presented. The genetic algorithm based optimization given in [71] generalizes these techniques for arbitrary register file sizes and auto-increment ranges while also incorporating MRs into memory layout construction.

\subsection{Code compaction}

Code compaction is typically executed as the last phase in code generation. At this point of time, all instructions required to implement a given application program have been generated, and the goal of code compaction is to schedule the generated sequential code into a minimum number of parallel machine instructions, or control steps, under the constraints imposed by the PDSP architecture and instruction set. Thus, code compaction is a variant of the resource constrained scheduling problem.

Input to the code compaction phase is usually a dependency graph $G=(V, E)$, whose nodes represent the instructions selected for a basic block, while edges denote scheduling precedences. There are three types of such precedences:

Data dependencies: Two instructions $I_{1}$ and $I_{2}$ are data dependent, if $I_{1}$ generates a value read by $I_{2}$. Thus, $I_{1}$ must be scheduled before $I_{2}$.

Anti dependencies: Two instructions $I_{1}$ and $I_{2}$ are anti dependent, if $I_{1}$ potentially overwrites a value still needed by $I_{2}$. Thus, $I_{1}$ must not be scheduled before $I_{2}$.

Output dependencies: Two instructions $I_{1}$ and $I_{2}$ are output dependent, if $I_{1}$ and $I_{2}$ write their results to the same location (register or memory cell). Thus, $I_{1}$ and $I_{2}$ must be scheduled in different control steps.

Additionally, incompatibility constraints $I_{1} \nsim I_{2}$ between instruction pairs $\left(I_{1}, I_{2}\right)$ have to be obeyed. These constraints arise either from processor resource limitations (e.g. only one multiplier available) or from the instruction format, which may 
prevent the parallel scheduling of instructions even without a resource conflict. In either case, if $I_{1} \not I_{2}$, then $I_{1}$ and $I_{2}$ must be scheduled in different control steps.

The code compaction problem has already been studied in the early eighties within the context of very long instruction word (VLIW) processors, showing a large degree of parallelism at the instruction level. A number of different compaction heuristics have been developed for VLIW machines [73]. However, even though PDSPs resemble VLIW machines to a certain extent, VLIW compaction techniques are not directly applicable to PDSPs. The reason is that instruction-level parallelism (ILP) is typically much more constrained in PDSPs than in VLIWs, because using very long instruction words for PDSPs would lead to extremely high code sizes. Furthermore, PDSP instruction sets frequently show alternative opcodes to perform a certain machine instruction.

As an example, consider the TI TMS320C25 instruction set. This PDSP offers instructions ADD and MPY to perform addition and multiplication. However, there is also a multiply-accumulate instruction MPYA, which performs both operations in parallel and thus faster. Instruction MPYA may be considered as an alternative opcode both for ADD and MPY, but its use is strongly context dependent. Only if an addition and a multiplication can be scheduled in parallel for a given dependency graph, MPYA may be used. Otherwise, using MPYA instead of either ADD or MPY could lead to an incorrect program behavior after compaction, because MPYA overwrites two registers (PR and ACCU), thus potentially causing undesired side effects.

In addition, code running onf PDSPs in most cases has to meet real-time constraints, which cannot be guaranteed by heuristics. Due to these special circumstances, DSP-specific code compaction techniques have been developed. In Timmer's approach [74], both resource and timing constraints are considered during code compaction. A bipartite graph is used to model possible assignments of instructions to control steps. In important feature of Timmer's technique is that timing constraints are exploited in order to quickly find exact solutions for compaction problem instances. The mobility of an instruction is the interval of control steps, to which an instruction may be assigned. Trivial bounds on mobility can be achieved by performing an ASAP/ALAP analysis on the dependency graph, which accounts for the earliest and the latest control step in which an instruction may be scheduled without violating dependencies. An additional execution interval analysis, based on both timing and resource constraints is performed to further restrict the mobility of instructions. The remaining mobility on the average is low, and a schedule meeting all constraints can be determined quickly by a branch-and-bound search.

Another DSP-specific code compaction technique was presented in [75], which also exploits the existence of alternative instruction opcodes. The code compaction problem is transformed into an Integer Linear Programming problem. In this formulation, a set of integer solution variables account for the detailed scheduling of instructions, while all precedences and constraints are modeled as linear equations and inequations on the solution variables. The Integer Linear Program is then solved optimally using a standard solver, such as "lp_solve" [76]. Since Integer Linear Programming is an exponential problem, the applicability of this technique is restricted to small to moderate size basic blocks, which however is sufficient in most practical cases.

In order to illustrate the impact of code compaction on code quality as well as its cooperation with other code generation phases, we use a small $\mathrm{C}$ program for complex number multiplication as an example.

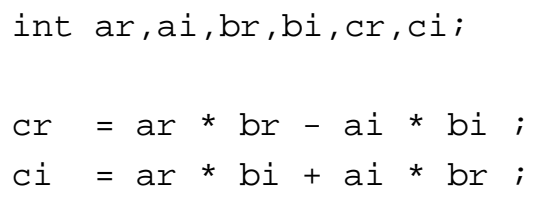

For the TI TMS320C25, the sequential assembly code, as generated by techniques mentioned in section 3.3, would be the following.

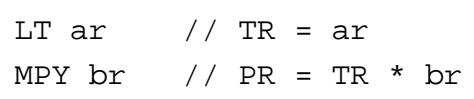




$$
\begin{array}{ll}
\mathrm{PAC} & / / \mathrm{ACCU}=\mathrm{PR} \\
\mathrm{LT} \text { ai } & / / \mathrm{TR}=\mathrm{ai} \\
\mathrm{MPY} \mathrm{bi} & / / \mathrm{PR}=\mathrm{TR} \text { bi } \\
\mathrm{SPAC} & / / \mathrm{ACCU}=\mathrm{ACCU}-\mathrm{PR} \\
\mathrm{SACL} \mathrm{Cr} & / / \mathrm{Cr}=\mathrm{ACCU} \\
\mathrm{LT} \text { ar } & / / \mathrm{TR}=\mathrm{ar} \\
\mathrm{MPY} \text { bi } & / / \mathrm{PR}=\mathrm{TR} \text { bi } \\
\mathrm{PAC} & / / \mathrm{ACCU}=\mathrm{PR} \\
\mathrm{LT} \text { ai } & / / \mathrm{TR}=\mathrm{ai} \\
\mathrm{MPY} \text { br } & / / \mathrm{PR}=\mathrm{TR} * \mathrm{br} \\
\mathrm{APAC} & / / \mathrm{ACCU}=\mathrm{ACCU}+\mathrm{PR} \\
\mathrm{SACL} \mathrm{Ci} & / / \mathrm{Ci}=\mathrm{ACCU}
\end{array}
$$

This sequential code shows the following (symbolic) variable access sequence:

$$
S=(a r, b r, a i, b i, c r, a r, b i, a i, b r, c i)
$$

Suppose, one address register AR is available for computing the memory addresses according to $S$. Then, the memory layout optimization mentioned in section 3.4 .2 would compute the following address mapping of the variables to the address space $[0,5]$.

\begin{tabular}{|l|l|}
\hline 0 & ci \\
1 & br \\
2 & ai \\
3 & bi \\
4 & cr \\
5 & ar \\
\hline
\end{tabular}

We can now insert the corresponding AGU operations into the sequential code and invoke code compaction. The resulting parallel assembly code makes use of parallelism both within the data path itself and with respect to parallel AGU operations (auto-increment and decrement).

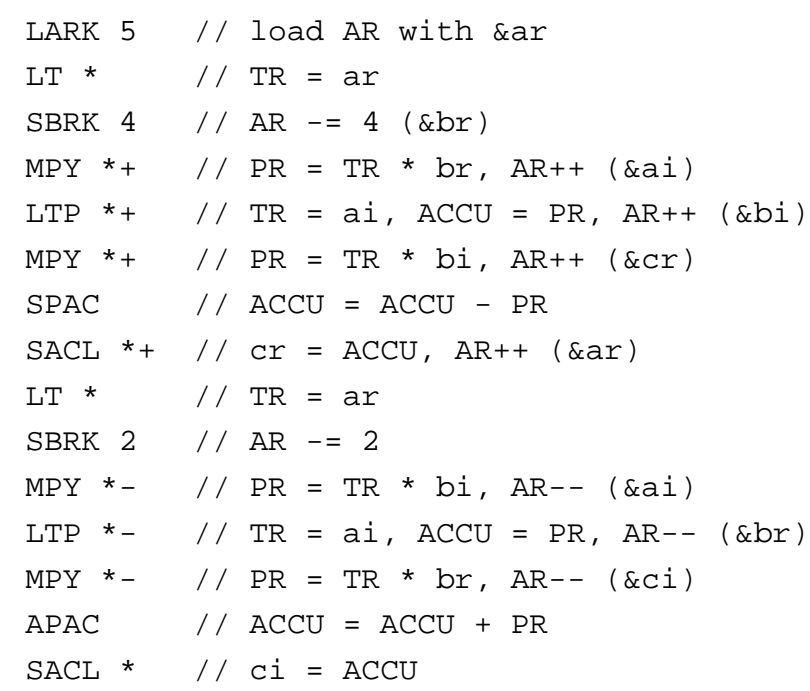

Even though address computations for the variables have been inserted, the resulting code is only one instruction larger than the original symbolic sequential code. This is achieved by a high utilization of zero-cost address computations (only 
two extra SBRK instructions) as well as parallel LTP instructions, which perform two data moves in parallel. This would not have been possible without memory layout optimization and code compaction.

\subsection{Phase coupling}

Even though code compaction is a powerful code optimization technique, only the direct coupling of sequential and parallel code generation phases can yield globally optimal results. Phase-coupled techniques frequently have to resort to heuristics due to extremely large search spaces. However, heuristics for phase-coupled code generation still may outperform exact techniques solving only parts of the code generation problem. In this section we therefore summarize important approaches to phase-coupled code generation for PDSPs.

Early work $[77,78]$ combined instruction scheduling with a data routing phase. In any step of scheduling, data routing performs detailed register allocation based on resource availability in accordance with a partial schedule constructed so far. In this way, the scheduling freedom (mobility) of instructions cannot be not obstructed by unfavorable register allocation decisions made earlier during code generation. However, significant effort has to be spent for avoidance of scheduling deadlocks, which restrict the applicability of such techniques to simple PDSP architectures.

Wilson's approach to phase coupled code generation [79] is also based on Integer Linear Programming. In his formulation, the complete search space, including register allocation, code selection, and code compaction is explored at once. While this approach permits the generation of provable optimal code for basic blocks, the high problem complexity also imposes heavy restrictions on applicability for realistic programs and PDSPs.

An alternative Integer Linear Programming formulation has been given in [80]. By better taking into account the detailed processor architecture, optimal code could be generated for small size examples for the TI TMS320C25 DSP.

A more practical phase coupling technique is Mutation Scheduling [81]. During instruction scheduling, a set of mutations is maintained for each program value. Each mutation represents an alternative implementation of value computation. For instance, mutations for a common subexpression in a DFG may include storing the CSE in some special purpose register or recomputing it multiple times. For other values, mutations are generated by application of algebraic rules like commutativity or associativity. In each scheduling step, the best mutation for each value to be scheduled is chosen. While Mutation Scheduling represents an "ideal" approach to phase coupling, its efficacy critically depends on the scheduling algorithm used as well as on the number of mutations considered for each value.

A constraint driven approach to phase-coupled code generation for PDSPs is presented in [82]. In that approach, alternatives with respect to code selection, register allocation, and scheduling are retained as long as possible during code generation. Restrictions imposed by the processor architecture are explicitly modeled in the form of constraints, which ensure correctness of the generated code. The implementation makes use of a constraint logic programming environment. For several examples it has been demonstrated that the quality of the generated code is equal to that of hand-written assembly code.

\subsection{Retargetable compilation}

As systems based on PDSPs mostly have to be very cost-efficient, a comparatively large number of different standard ("offthe-shelf") PDSPs are available on the semiconductor market at the same time. From this variety, a PDSP user may select that processor architecture which matches his requirements at minimum costs. In spite of the large variety of standard DSPs, however, it is still unlikely that a customer will find a processor ideally matching one given application. In particular, using standard processors in the form of cores (layout macro cells) for systems-on-a-chip may lead to a waste of silicon area. For mobile applications, also the electrical power consumed by a standard processor may be too high.

As a consequence, there is a trend towards the use of a new class of PDSPs, called application specific signal processors (ASSPs). The architecture of such ASSPs is still programmable, but is customized for restricted application areas. A wellknown example is the EPICS architecture [83]. A number of further ASSPs are mentioned in [52]. 
The increasing use of ASSPs for implementing embedded DSP systems leads to an even larger variety of PDSPs. While the code optimization techniques mentioned in the previous sections help to improve the practical applicability of compilers for DSP software development, they do not answer the question: Who will write compilers for all these different PDSP architectures? Developing a compiler for each new ASSP, possibly having a low production volume and product lifetime, is not economically feasible. Still, the use of compilers for ASSPs instead of assembly programming is still highly desirable.

Therefore, researchers have looked at technology for developing retargetable compilers. Such compilers are not restricted to generating code for a single target processor, but are sufficiently flexible to be reused for a whole class of PDSPs. More specifically, we call a compiler retargetable, if adapting the compiler to a new target processor does not involve rewriting a large part of the compiler source code. This can be achieved by using external processor models. While in a classical, target-specific compiler the processor model is hard-coded in the compiler source code, a retargetable compiler can read an external processor model as an additional input specified by the user and generate code for the target processor specified by the model.

\subsubsection{The RECORD compiler system}

An example of a retargetable compiler for PDSPs is the RECORD system [84], a coarse overview of which is given in fig. 25. In RECORD, processor models are given in the hardware description language (HDL) MIMOLA, which resembles structural VHDL. A MIMOLA processor model captures the register transfer level structure of a PDSPs, including controller, data path, and address generation units. Alternatively, the pure instruction set can be described, while hiding the internal structure. Using HDL models is a natural way of describing processor hardware, with a large amount of modeling flexibility. Furthermore, the use of HDL models reduces the number of different processor models required during the design process, since HDL models can be used also for hardware synthesis and simulation.

Sequential code generation in RECORD is based on the data flow tree (DFT) model explained in section 3.3.1. The source program, given in the programming language DFL, is first transformed into an intermediate representation, consisting of DFTs. The code generator is automatically generated from the HDL processor model by means of the iburg tool [59]. Since iburg requires a tree grammar model of the target instruction set, some preprocessing of the HDL model is necessary. RECORD uses an instruction set extraction phase to transform the structural HDL model into an internal model of the machine instruction set. This internal model captures the behavior of available machine instructions as well as the constraints on instruction-level parallelism.

During sequential code generation, the code generator generated by means of iburg is used to map DFTs into target specific machine code. While mapping, RECORD exploits algebraic rules like commutativity and associativity of operators to increase code quality. The resulting sequential assembly code is further optimized by means of memory access optimization (section 3.4) and code compaction (section 3.5). An experimental evaluation for the TI TMS320C25 DSP showed, that thanks to these optimizations RECORD on the average generates significantly denser code than a commercial target specific compiler, however at the expense of lower compilation speed. Furthermore, RECORD is easily retargetable to different processor architectures. If a HDL model is available, then generation of processor specific compiler components typically takes less than one workstation CPU minute. This short turnaround time permits to use a retargetable compiler also for quickly exploring different architectural options for an ASSP, e.g., with respect to the number of functional units, register file sizes, or interconnect structure.

\subsubsection{Further retargetable compilers}

A widespread example for a retargetable compiler is the GNU compiler "gcc" [85]. Since gcc has been mainly designed for CISC and RISC processor architectures, it is based on the assumption of regular processor architectures and thus is hardly applicable to PDSPs. 
The MSSQ compiler [86] has been an early approach to retargetable compilation based on HDL models, however without specific optimizations for PDSPs.

In the CodeSyn compiler [57], specifically designed for ASSPs, the target processor is heterogeneously described by the set of available instruction patterns, a graph model representing the data path, and a resource classification that accounts for special purpose registers.

The CHESS compiler [87] uses a specific language called nML for describing target processor architectures. It generates code for a specific ASSP architectural style and therefore employs special code generation and optimization techniques [88]. The nML language has also been used in a retargetable compiler project at Cadence [89].

Several code optimizations mentioned in this paper [61, 62, 60, 63] have been implemented in the SPAM compiler at Princeton University and MIT. Although SPAM can be classified as a retargetable compiler, it is more based on exchangeable software modules performing specific optimization instead of an external target processor model.

Another approach to retargetable code generation for PDSPs is the AVIV compiler [90], which uses a special language (ISDL [91]) for modeling VLIW-like processor architectures.

As compilers for standard DSPs and ASSPs become more important and retargetable compiler technology gets more mature, several companies have started to sell commercial retargetable compilers with special emphasis on PDSPs. Examples are the CoSy compiler development system by ACE, the commercial version of the CHESS compiler, as well as Archelon's retargetable compiler system. Detailed information about these recent software products is available on the World Wide Web $[92,93,94]$.

\section{Conclusions}

This paper has reviewed that state of the art in front- and back-end design automation technology for DSP software implementation. We have motivated a design flow that begins with a high-level, hierarchical block diagram specification; synthesizes a C-language application program or subsystem from this specification; and then compiles the $\mathrm{C}$ program into optimized machine code for the given target processor. We have reviewed several useful computational models that provide efficient semantics for the block diagram specifications at the front end of this design flow, We then examined the vast space of implementation trade-offs one encounters when synthesizing software from these computational models, in particular from the closely-related synchronous dataflow (SDF) and scalable synchronous dataflow (SSDF) models, which can be viewed as key "common denominators" of the other models. Subsequently, we examined a variety of useful software synthesis techniques that address important subsets of and prioritizations of relevant optimization metrics.

Complementary to software synthesis issues, we have outlined the state-of-the-art in compilation of efficient machine code from application source programs. Taking the step from assembly-level to C-level programming of DSPs demands for special code generation techniques beyond the scope of classical compiler technology. In particular, this concerns code generation, memory access optimization, and exploitation of instruction-level parallelism. Recently, also the problem of tightly coupling these different compilation phases in order to generated very efficient code has gained significant research interest. In addition, we have motivated the use of retargetable compilers, which are important for programming applicationspecific DSPs.

In our overview, we have highlighted useful directions for further study. A particularly interesting and promising direction, which remains largely unexplored, is the investigation of the interaction between software synthesis and code generation - that is, the development of synthesis techniques that explicitly aid the code generation process, and code generation techniques that incorporate high-level application structure that is exposed during synthesis. 


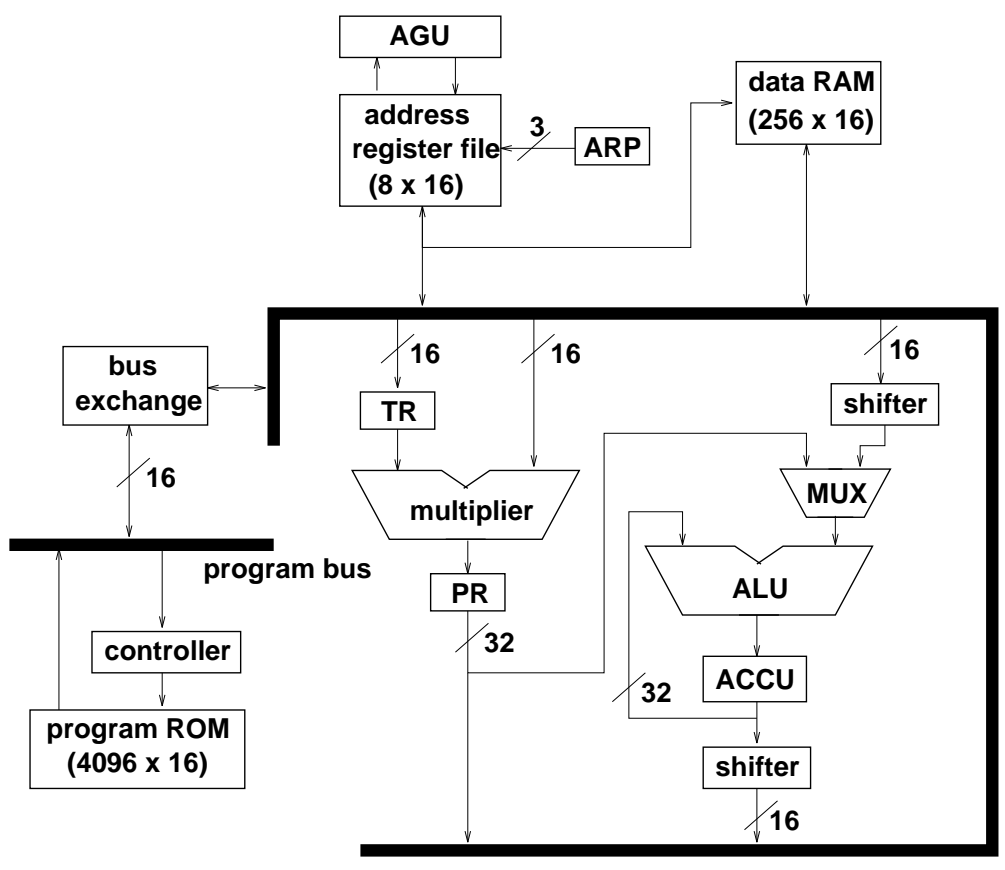

data bus

Figure 1: Simplified architecture of Texas Instruments TMS320C25 DSP 


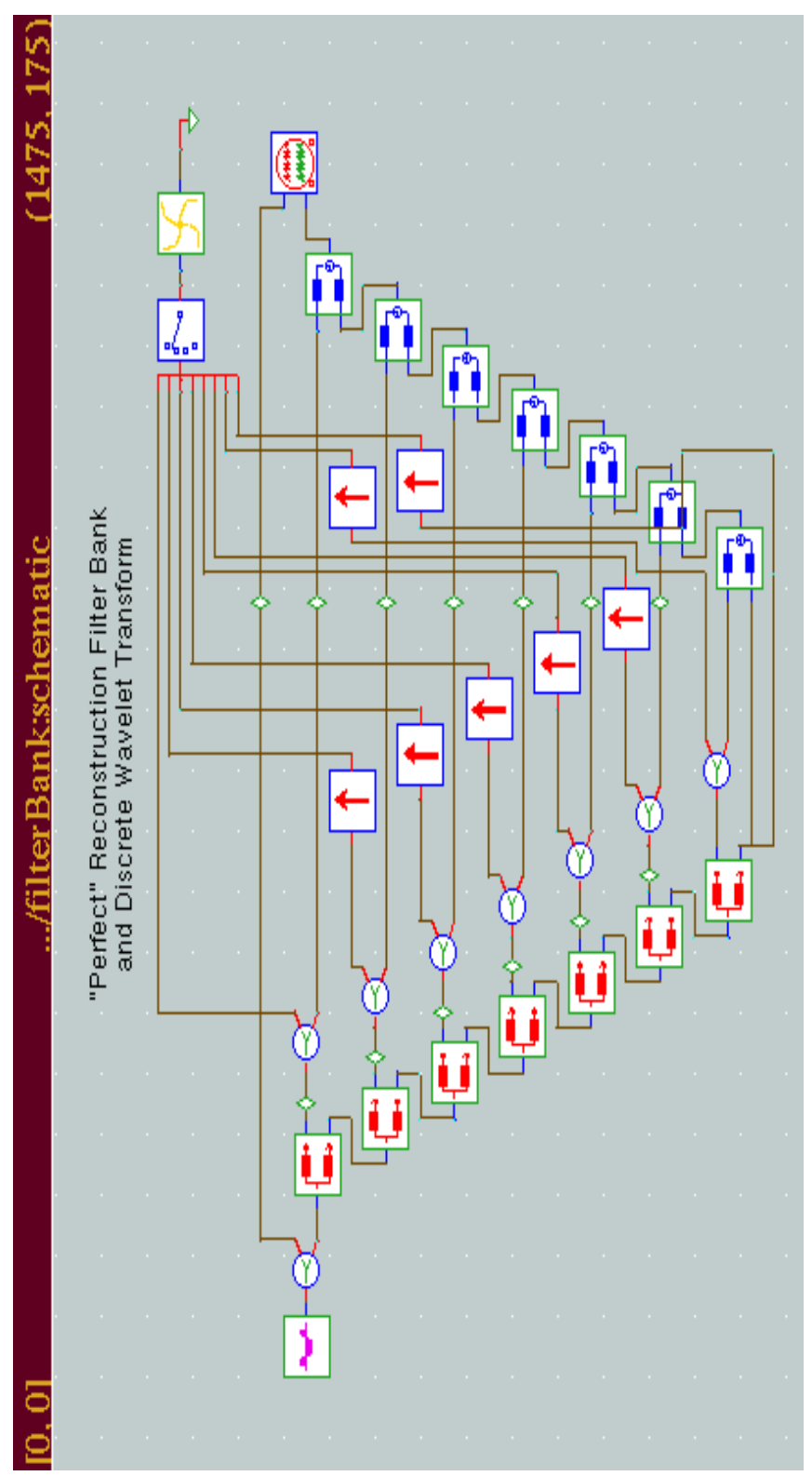

Figure 2: The top-level block diagram specification of a discrete wavelet transform application implemented in Ptolemy [7]. 


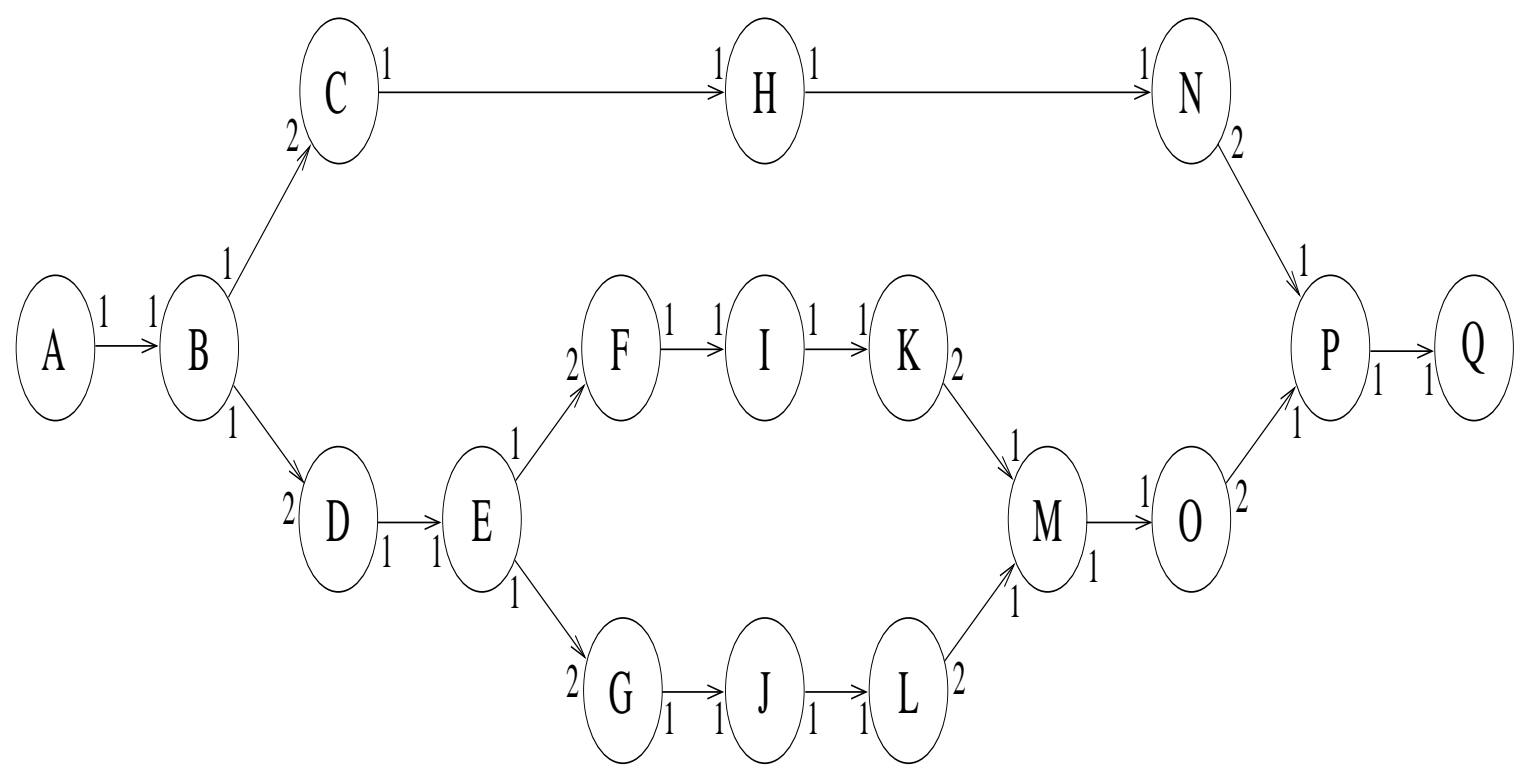

Figure 3: An illustration of an explicit SDF specification.

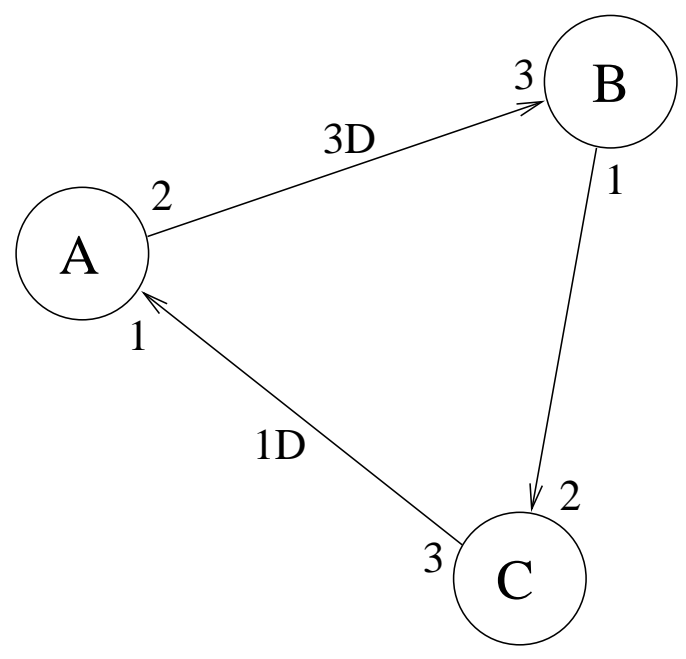

Figure 4: A deadlocked SDF graph.

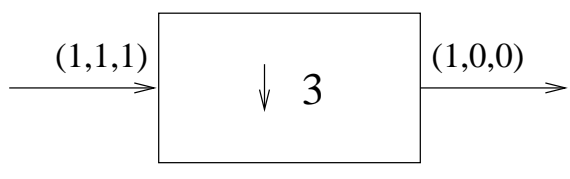

(a)

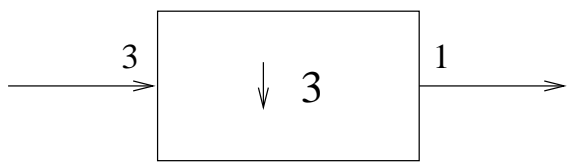

(b)

Figure 5: CSDF and SDF versions of a downsampler block. 


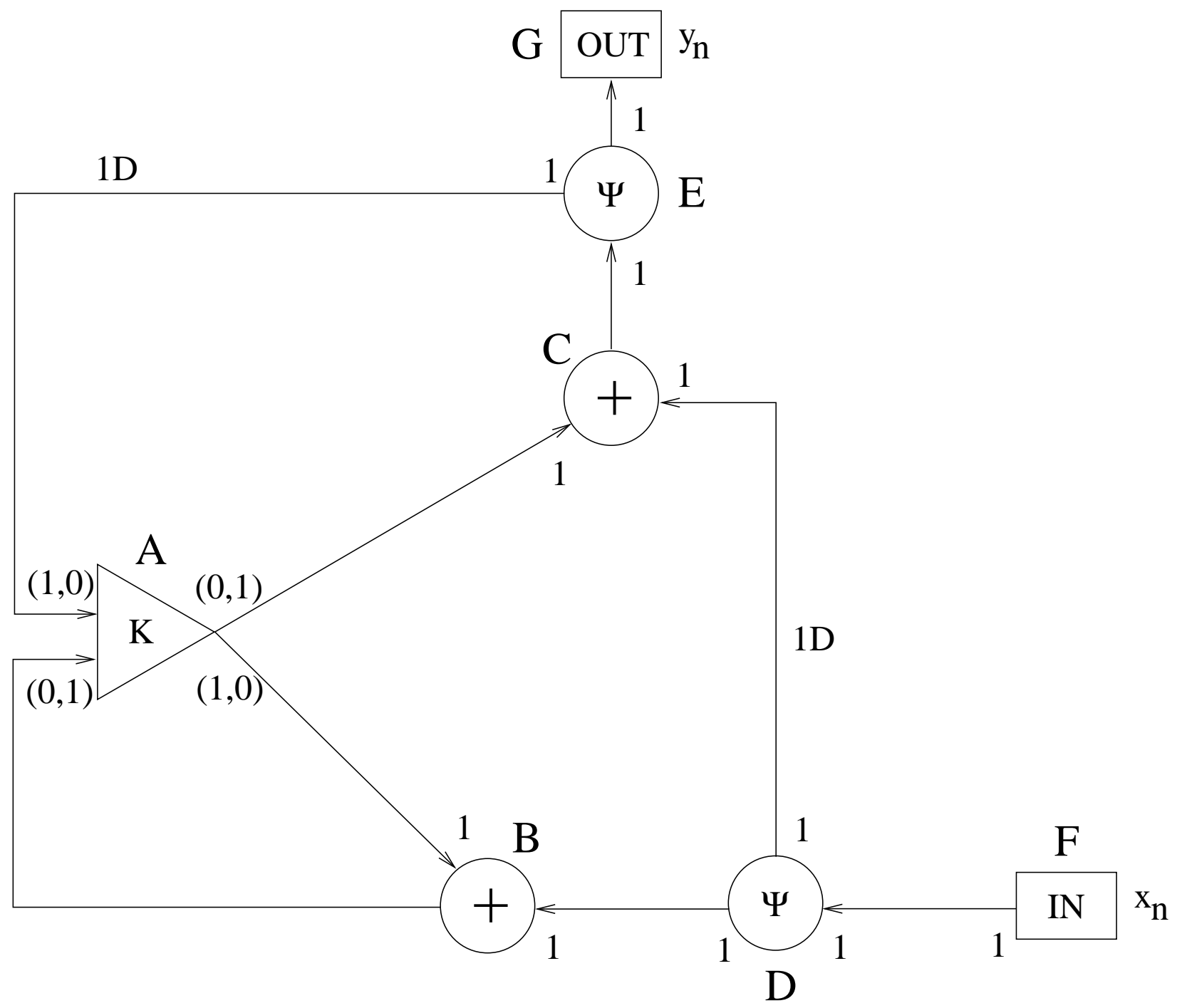

$$
\begin{aligned}
\mathrm{y}_{\mathrm{n}} & =\mathrm{k}^{2} \mathrm{y}_{\mathrm{n}-1}+\mathrm{kx}_{\mathrm{n}}+\mathrm{x}_{\mathrm{n}-1} \\
& =\mathrm{k}\left(\mathrm{ky}_{\mathrm{n}}+\mathrm{x}_{\mathrm{n}}\right)+\mathrm{x}_{\mathrm{n}-1}
\end{aligned}
$$

\section{valid schedule: $\mathrm{A}_{1} \mathrm{FDBA}_{2} \mathrm{CEG}$}

Figure 6: An example that illustrates the compact modeling of resource sharing using CSDF. The actor labeled $\Psi$ denotes a dataflow fork, which simply replicates its input tokens on all of its output edges. The lower portion of the figure gives a valid schedule for this CSDF specification. Here, $A_{1}$ and $A_{2}$ denote the first and second phases of the CSDF actor $A$. 


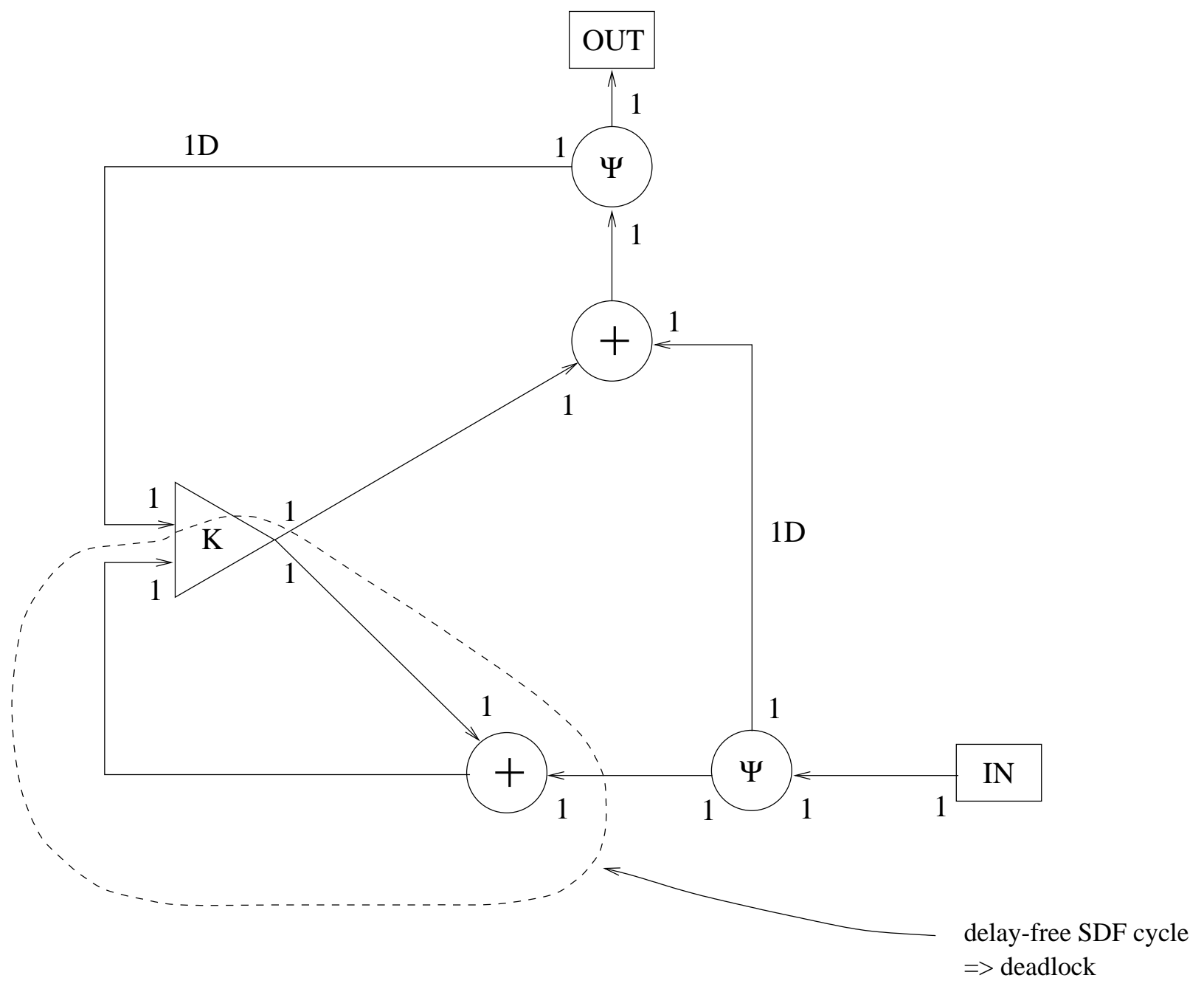

Figure 7: The SDF version of the specification in fig. 6. 


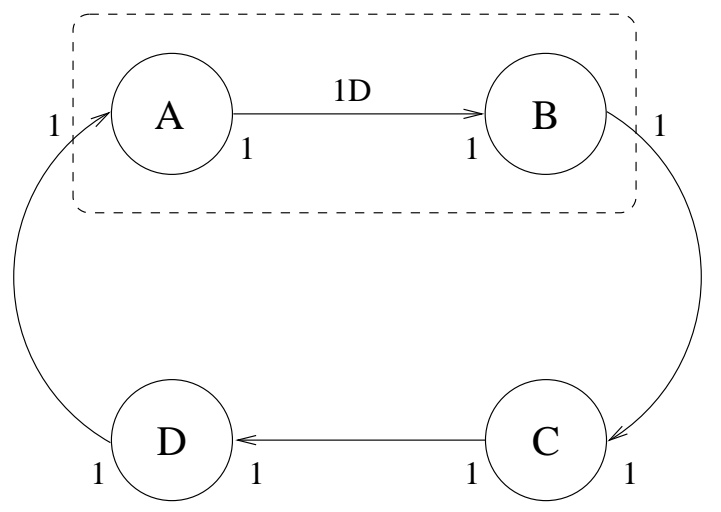

(a)

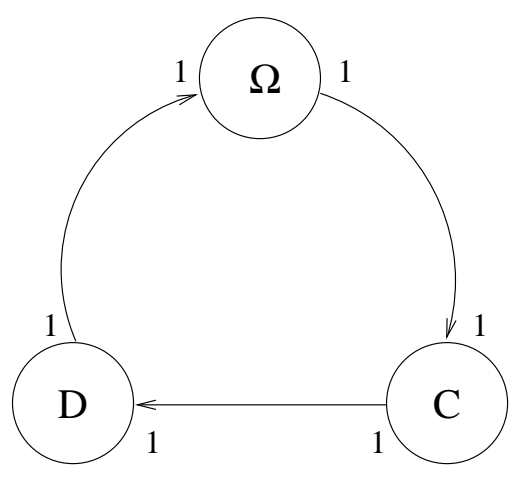

(b)

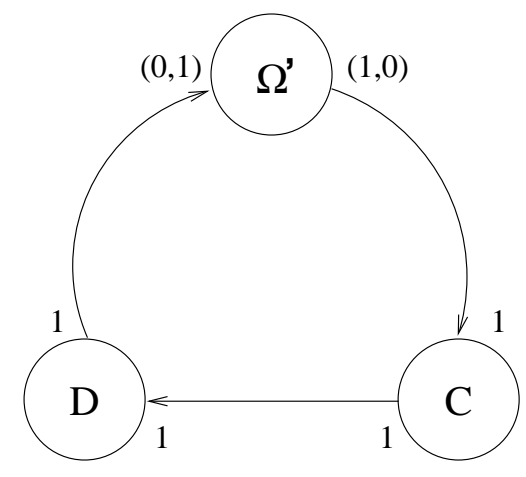

$\Omega_{1}^{\prime}: \mathrm{B}$

$\Omega_{2}^{\prime}: \mathrm{A}$

schedule: $\Omega_{1}^{\prime} \mathrm{CD} \Omega_{2}^{\prime}$

(c)

Figure 8: An example that illustrates the utility of cyclo-static dataflow in constructing hierarchical specifications. Grouping the actors $A$ and $B$ into the hierarchical SDF actor $\Omega$, as shown in (b), results in a deadlocked SDF graph. In contrast, an appropriate CSDF model of the hierarchical grouping, illustrated in (c), avoids deadlock. The two phases of the hierarchical $\mathrm{CSDF}$ actor $\Omega^{\prime}$ in (c) are specified in the lower right corner of the figure along with a valid schedule for the CSDF specification. 


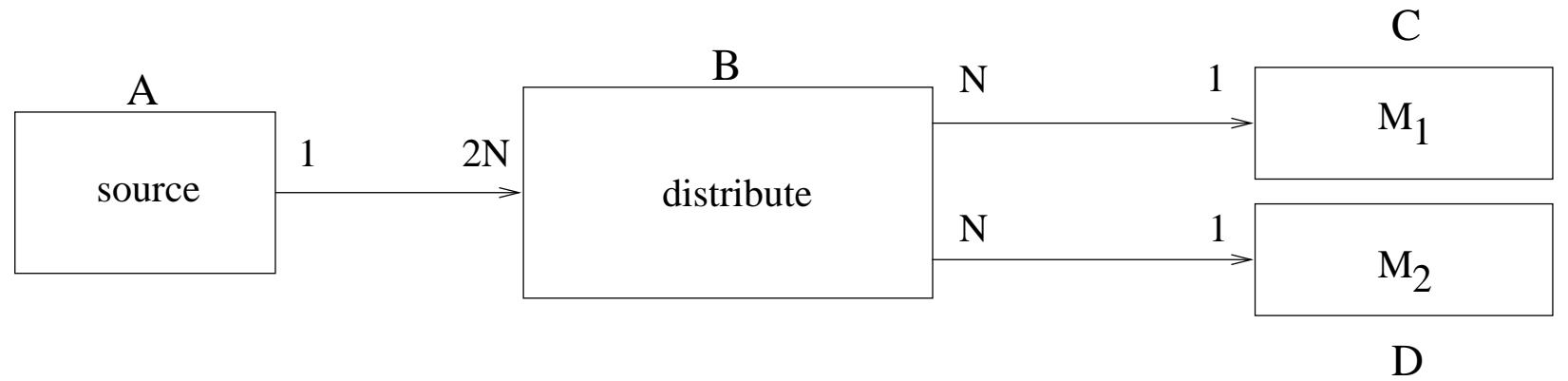

(a)

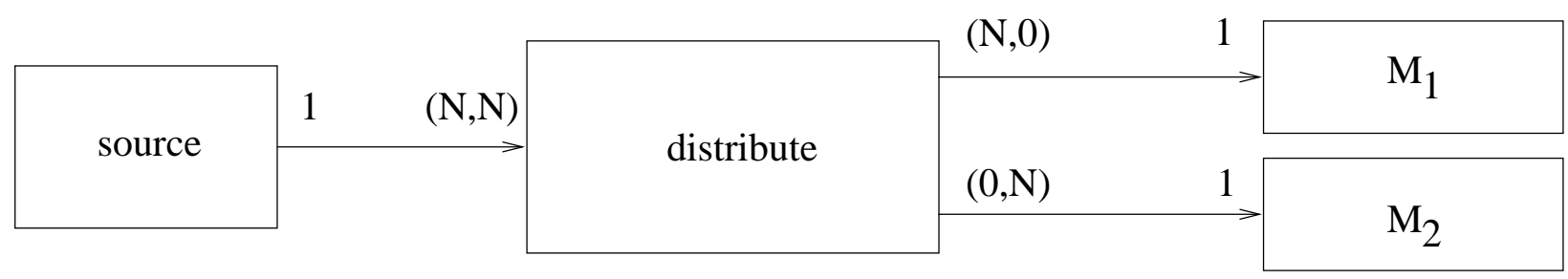

(b)

Figure 9: An example of the use of CSDF to decrease buffering requirements. 


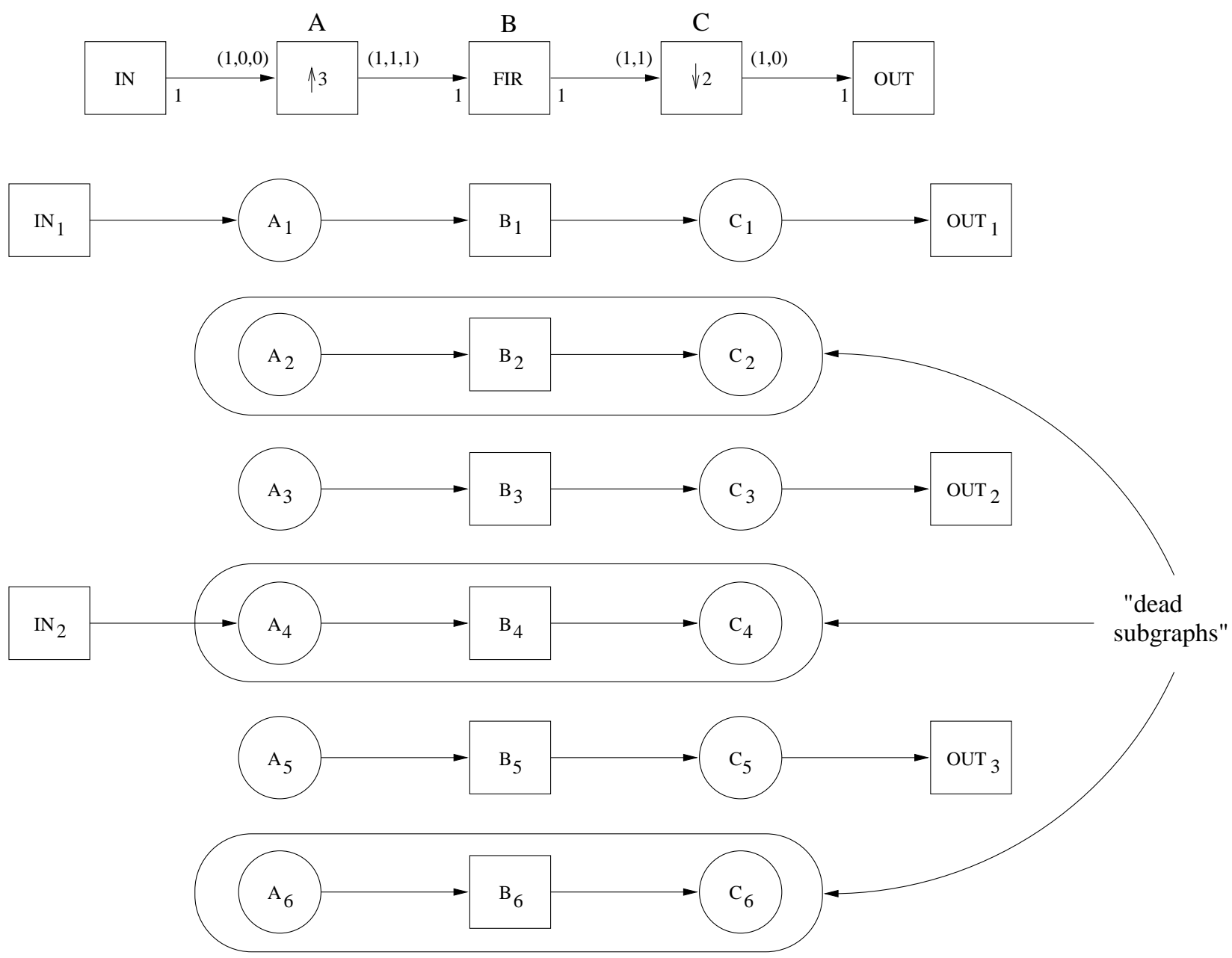

Figure 10: An example of efficient dead code elimination using CSDF.

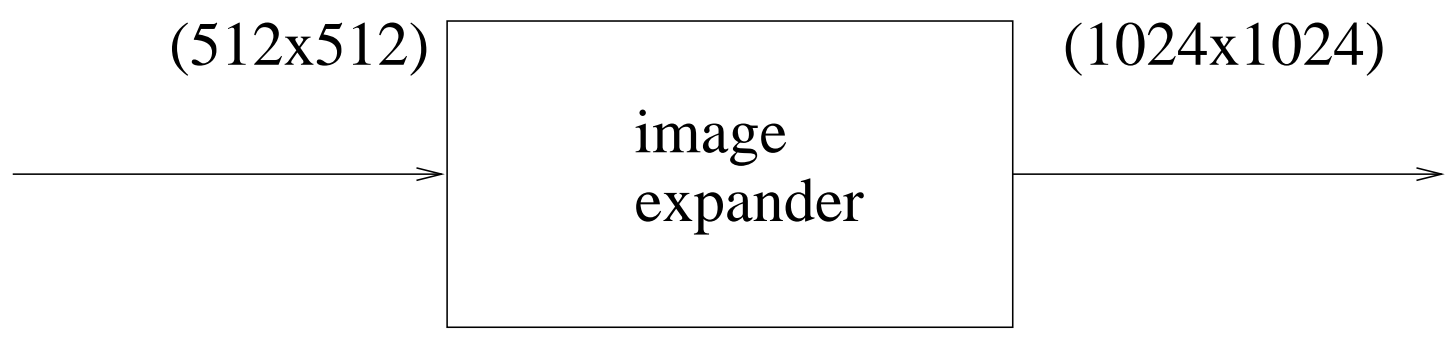

Figure 11: An example of an MDSDF actor.

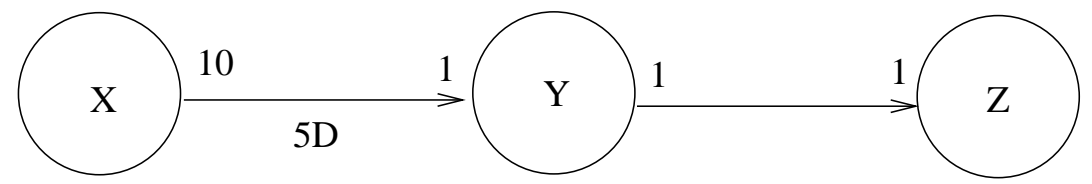

Figure 12: A simple example that we use to illustrate trade-offs involved in compiling SDF specifications. 


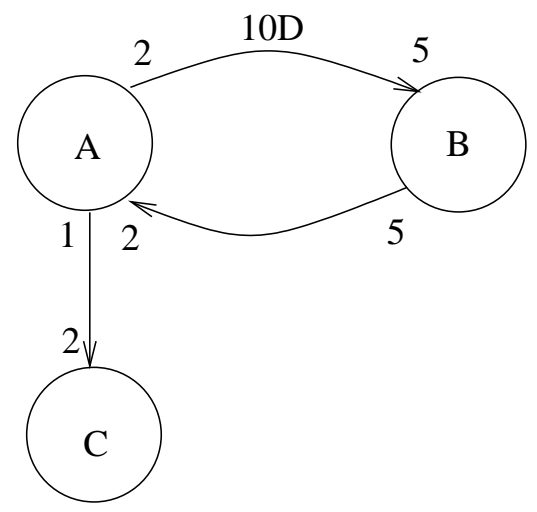

Figure 13: An example that we use to illustrate the $N_{a c t}$ metric.

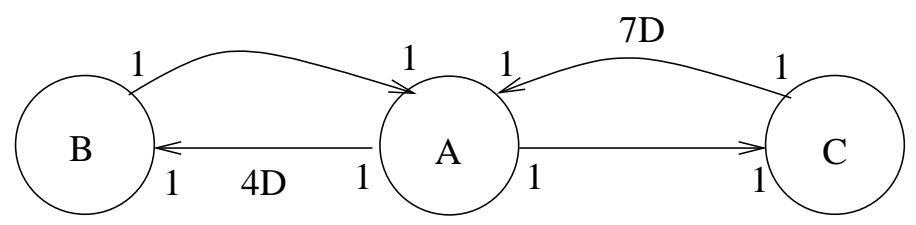

Figure 14: This example illustrates that minimizing actor activations does not imply minimizing actor appearances. 


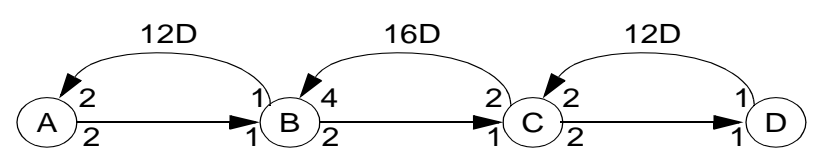

(a)

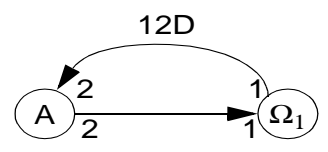

(b)

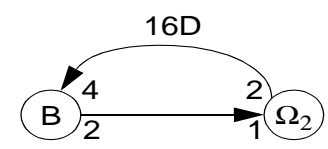

(c)

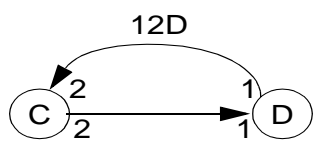

(d)

101

Figure 15: An illustration of a complete hierarchization. 


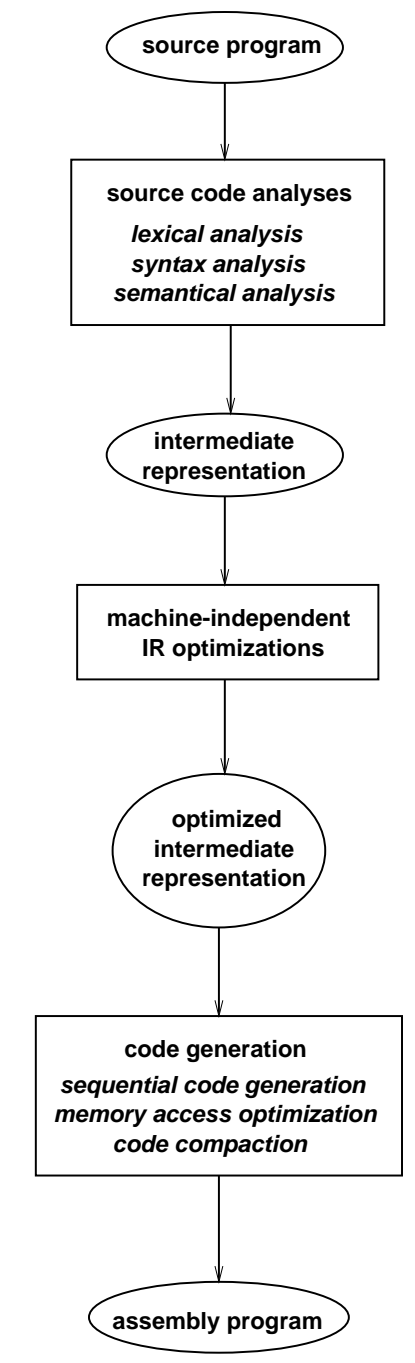

Figure 16: Compilation phases

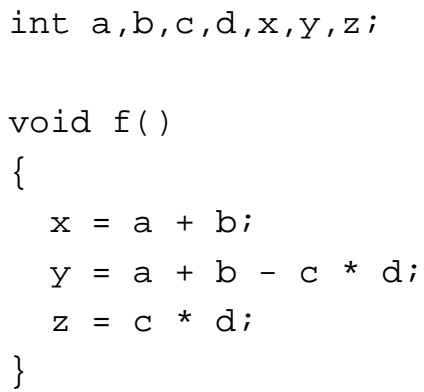

Figure 17: Example C source code 


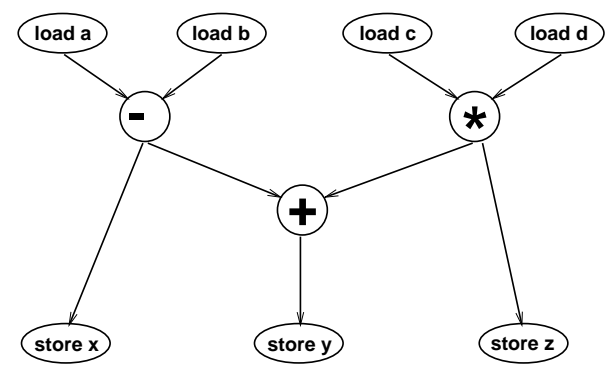

Figure 18: DFG representation of code from fig. 17

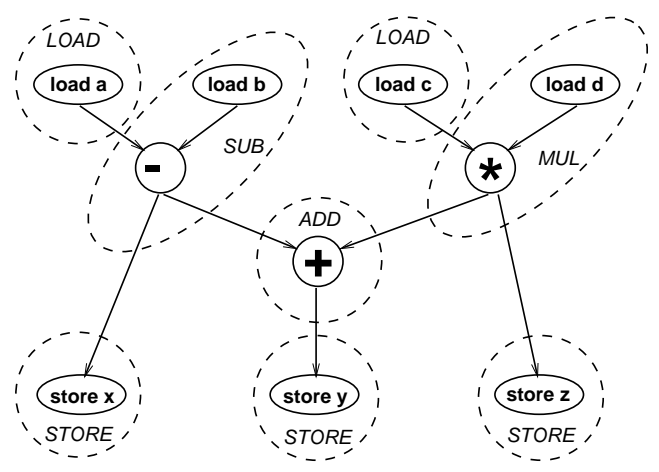

Figure 19: DFG from fig. 18 covered by instruction patterns

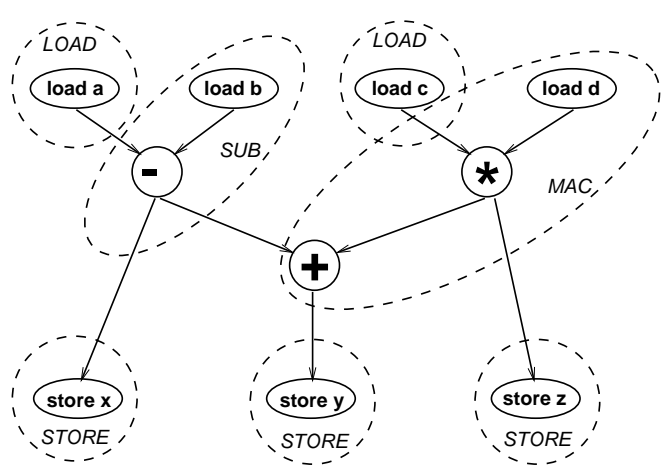

Figure 20: Using MAC for DFG covering

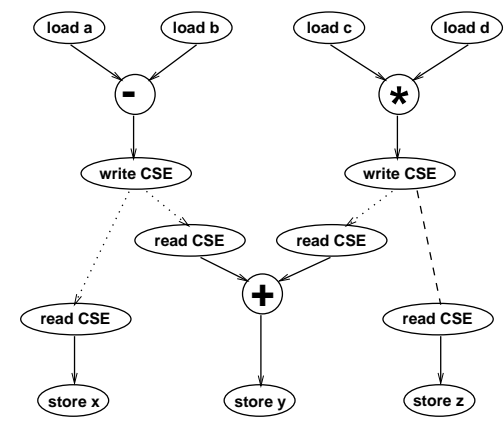

Figure 21: Decomposition of a DFG into DFTs 


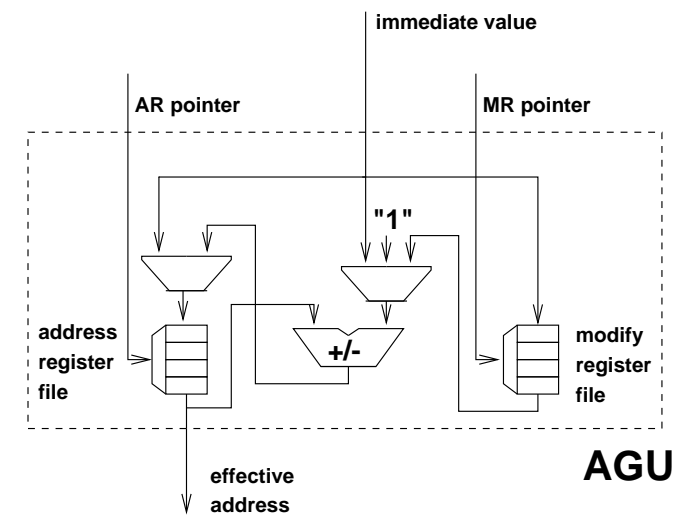

Figure 22: Address generation unit

\begin{tabular}{|c|c|c|c|c|c|c|c|c|c|c|}
\hline & & $\begin{array}{l}\text { LOAD AR, } 1 \\
\text { AR }+=2 \\
A R=3\end{array}$ & $\begin{array}{l}\text { b } \\
\text { d } \\
\text { a }\end{array}$ & & & \multirow{6}{*}{\multicolumn{2}{|c|}{$\begin{array}{ll}\text { LOAD AR, } 3 \text { b } \\
\text { AR -- } & \text { d } \\
\text { AR -- } & \text { a } \\
\text { AR -- } & \text { c } \\
\text { AR += 2 } & \text { d } \\
\text { AR -- } & \text { a } \\
\text { AR -- } & \text { C } \\
\text { AR += 3 } & \text { b } \\
\text { AR -= 2 } & \text { a } \\
\text { AR ++ } & \text { d } \\
\text { AR -- } & \text { a } \\
\text { AR -- } & \text { c } \\
\text { AR += 2 } & \text { d }\end{array}$}} & \multirow{5}{*}{$\begin{array}{l}0 \\
1 \\
2 \\
3\end{array}$} & \multirow{4}{*}{$\begin{array}{l}\text { c } \\
a \\
d\end{array}$} & \multirow{6}{*}{$\begin{array}{l}\text { LOAD AR, } 3 \\
\text { AR -- } \\
\text { AR -- } \\
\text { AR -- } \\
\text { LOAD MR, } 2 \\
\text { AR += MR } \\
\text { AR -- } \\
\text { AR -- } \\
\text { AR += 3 } \\
\text { AR -= MR } \\
\text { AR ++ } \\
\text { AR -- } \\
\text { AR -- } \\
\text { AR += MR }\end{array}$} \\
\hline 0 & $\mathbf{a}$ & $\begin{array}{l}\mathrm{AR}+=2 \\
\mathrm{AR}++\end{array}$ & d & 0 & c & & & & & \\
\hline 1 & b & $A R=3$ & a & 1 & a & & & & & \\
\hline 2 & c & $A R+=2$ & c & 2 & d & & & & & \\
\hline 3 & d & AR -- & b & 3 & b & & & & b & \\
\hline & : 9 & $\begin{array}{l}\text { AR -- } \\
A R+=3 \\
A R=3 \\
A R+=2 \\
A R++\end{array}$ & $\begin{array}{l}\text { d } \\
\text { a } \\
\text { c } \\
\text { d }\end{array}$ & $\cos$ & & & & $\cos$ & & \\
\hline & & ) & & & & )) & & & & \\
\hline
\end{tabular}

Figure 23: Alternative memory layouts and AGU operation sequences
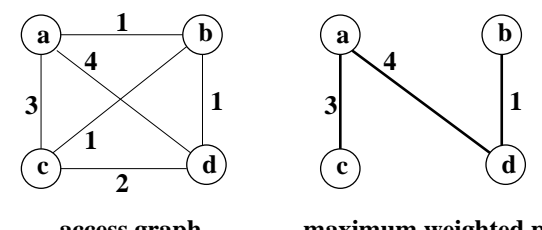

access graph

maximum weighted path

Figure 24: Access graph model and maximum weighted path 


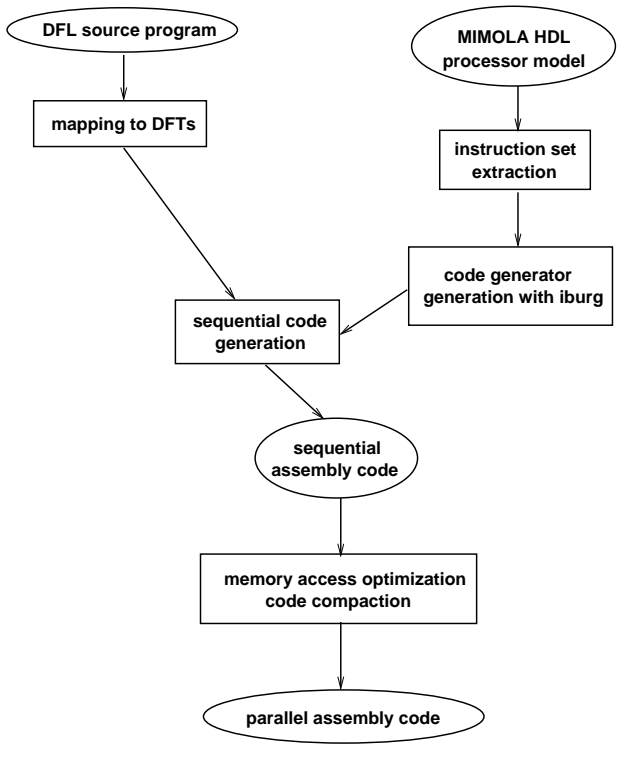

Figure 25: Coarse architecture of the RECORD system 


\section{References}

[1] The Design and Implementation of Signal Processing Systems Technical Committee. VLSI design and implementation fuels the signal processing revolution. IEEE Signal Processing Magazine, 15(1):22-37, January 1998.

[2] P. Lapsley, J. Bier, A. Shoham, and E. A. Lee. DSP Processor Fundamentals. Berkeley Design Technology, Inc., 1994.

[3] E. A. Lee. Programmable DSP architectures — Part I. IEEE ASSP Magazine, 5(4), October 1988.

[4] E. A. Lee. Programmable DSP architectures — Part II. IEEE ASSP Magazine, 6(1), January 1988.

[5] P. Marwedel and G. Goossens, editors. Code Generation for Embedded Processors. Kluwer Academic Publishers, 1995.

[6] V. Zivojnovic, H. Schraut, M. Willems, and H. Meyr. DSPs, GPPs, and multimedia applications - an evaulation using DSPstone. In Proceedings of the International Conference on Signal Processing Applications and Technology, November 1995.

[7] J. T. Buck, S. Ha, E. A. Lee, and D. G. Messerschmitt. Ptolemy: A framework for simulating and prototyping heterogeneous systems. International Journal of Computer Simulation, January 1994.

[8] P. P. Vaidyanathan. Multirate Systems and Filter Banks. Prentice Hall, 1993.

[9] E. A. Lee and D. G. Messerschmitt. Synchronous dataflow. Proceedings of the IEEE, 75(9):1235-1245, September 1987.

[10] E. A. Lee. Consistency in dataflow graphs. IEEE Transactions on Parallel and Distributed Systems, 2(2), April 1991.

[11] S. S. Bhattacharyya, P. K. Murthy, and E. A. Lee. Software Synthesis from Dataflow Graphs. Kluwer Academic Publishers, 1996.

[12] S. Ritz, M. Willems, and H. Meyr. Scheduling for optimum data memory compaction in block diagram oriented software synthesis. In Proceedings of the International Conference on Acoustics, Speech, and Signal Processing, May 1995.

[13] E. A. Lee and D. G. Messerschmitt. Static scheduling of synchronous dataflow programs for digital signal processing. IEEE Transactions on Computers, February 1987.

[14] E. A. Lee, W. H. Ho, E. Goei, J. Bier, and S. S. Bhattacharyya. Gabriel: A design environment for DSP. IEEE Transactions on Acoustics, Speech, and Signal Processing, 37(11), November 1989.

[15] D. R. O'Hallaron. The ASSIGN parallel program generator. Technical report, School of Computer Science, Carnegie Mellon University, May 1991.

[16] G. Bilsen, M. Engels, R. Lauwereins, and J. A. Peperstraete. Cyclo-static data flow. In Proceedings of the International Conference on Acoustics, Speech, and Signal Processing, pages 3255-3258, May 1995.

[17] G. Bilsen, M. Engels, R. Lauwereins, and J. A. Peperstraete. Cyclo-static dataflow. IEEE Transactions on Signal Processing, 44(2):397-408, February 1996.

[18] G. De Micheli. Synthesis and Optimization of Digital Circuits. McGraw-Hill, 1994.

[19] T. M. Parks, J. L. Pino, and E. A. Lee. A comparison of synchronous and cyclo-static dataflow. In Proceedings of the IEEE Asilomar Conference on Signals, Systems, and Computers, November 1995. 
[20] S. Ritz, M. Pankert, and H. Meyr. Optimum vectorization of scalable synchronous dataflow graphs. In Proceedings of the International Conference on Application Specific Array Processors, October 1993.

[21] S. Ritz, M. Pankert, and H. Meyr. High level software synthesis for signal processing systems. In Proceedings of the International Conference on Application Specific Array Processors, August 1992.

[22] E. A. Lee. Representing and exploiting data parallelism using multidimensional dataflow diagrams. In Proceedings of the International Conference on Acoustics, Speech, and Signal Processing, pages 453-456, April 1993.

[23] P. K. Murthy and E. A. Lee. An extension of multidimensional synchronous dataflow to handle arbitrary sampling lattices. In Proceedings of the International Conference on Acoustics, Speech, and Signal Processing, pages 33063309, May 1996.

[24] G. R. Gao, R. Govindarajan, and P. Panangaden. Well-behaved programs for DSP computation. In Proceedings of the International Conference on Acoustics, Speech, and Signal Processing, March 1992.

[25] J. T. Buck and E. A. Lee. Scheduling dynamic dataflow graphs using the token flow model. In Proceedings of the International Conference on Acoustics, Speech, and Signal Processing, April 1993.

[26] J. T. Buck. Scheduling Dynamic Dataflow Graphs with Bounded Memory using the Token Flow Model. PhD thesis, Department of Electrical Engineering and Computer Sciences, University of California at Berkeley, September 1993.

[27] J. T. Buck. Static scheduling and code generation from dynamic dataflow graphs with integer-valued control systems. In Proceedings of the IEEE Asilomar Conference on Signals, Systems, and Computers, October 1994.

[28] S. S. Bhattacharyya, P. K. Murthy, and E. A. Lee. Optimal parenthesization of lexical orderings for DSP block diagrams. In Proceedings of the International Workshop on VLSI Signal Processing. IEEE press, October 1995. Sakai, Osaka, Japan.

[29] M. Ade, R. Lauwereins, and J. A. Peperstraete. Buffer memory requirements in DSP applications. In Proceedings of the IEEE Workshop on Rapid System Prototyping, pages 198-123, June 1994.

[30] M. Ade, R. Lauwereins, and J.A. Peperstraete. Data memory minimisation for synchronous data flow graphs emulated on DSP-FPGA targets. In Proceedings of the Design Automation Conference, pages 64-69, June 1994.

[31] M. Cubric and P. Panangaden. Minimal memory schedules for dataflow networks. In CONCUR '93, August 1993.

[32] R. Govindarajan, G. R. Gao, and P. Desai. Minimizing memory requirements in rate-optimal schedules. In Proceedings of the International Conference on Application Specific Array Processors, August 1994.

[33] S. How. Code generation for multirate DSP systems in gabriel. Master's thesis, Department of Electrical Engineering and Computer Sciences, University of California at Berkeley, May 1990.

[34] S. S. Bhattacharyya, P. K. Murthy, and E. A. Lee. Synthesis of embedded software from synchronous dataflow specifications. Journal of VLSI Signal Processing Systems, 21(2):151-166, June 1999.

[35] S. S. Bhattacharyya, J. T. Buck, S. Ha, and E. A. Lee. A scheduling framework for minimizing memory requirements of multirate DSP systems represented as dataflow graphs. In Proceedings of the International Workshop on VLSI Signal Processing, October 1993. Veldhoven, The Netherlands.

[36] S. S. Bhattacharyya, J. T. Buck, S. Ha, and E. A. Lee. Generating compact code from dataflow specifications of multirate signal processing algorithms. IEEE Transactions on Circuits and Systems - I: Fundamental Theory and Applications, 42(3):138-150, March 1995. 
[37] S. S. Bhattacharyya, P. K. Murthy, and E. A. Lee. APGAN and RPMC: Complementary heuristics for translating DSP block diagrams into efficient software implementations. Journal of Design Automation for Embedded Systems, January 1997.

[38] P. K. Murthy, S. S. Bhattacharyya, and E. A. Lee. Joint minimization of code and data for synchronous dataflow programs. Journal of Formal Methods in System Design, 11(1):41-70, July 1997.

[39] J. L. Pino, S. S. Bhattacharyya, and E. A. Lee. A hierarchical multiprocessor scheduling system for DSP applications. In Proceedings of the IEEE Asilomar Conference on Signals, Systems, and Computers, November 1995.

[40] P. K. Murthy and S. S. Bhattacharyya. Shared memory implementations of synchronous dataflow specifications using lifetime analysis techniques. Technical Report UMIACS-TR-99-32, Institute for Advanced Computer Studies, University of Maryland at College Park, June 1999.

[41] P. K. Murthy and S. S. Bhattacharyya. A buffer merging technique for reducing memory requirements of synchronous dataflow specifications. In Proceedings of the International Symposium on Systems Synthesis, 1999. San Jose, California, to appear.

[42] E. Zitzler, J. Teich, and S. S. Bhattacharyya. Optimized software synthesis for DSP using randomization techniques. Technical report, Computer Engineering and Communication Networks Laboratory, Swiss Federal Institute of Technology, Zurich, July 1999. Revised version of teic1998x1.

[43] J. Teich, E. Zitzler, and S. S. Bhattacharyya. Optimized software synthesis for digital signal processing algorithms an evolutionary approach. In Proceedings of the IEEE Workshop on Signal Processing Systems, October 1998. Boston, Massachusetts.

[44] E. Zitzler, J. Teich, and S. S. Bhattacharyya. Evolutionary algorithms for the synthesis of embedded software. IEEE Transactions on Very Large Scale Integration (VLSI) Systems, 1999. Accepted for publication; to appear.

[45] T. Back, U. Hammel, and H-P Schwefel. Evolutionary computation: Comments on the history and current state. IEEE Transactions on Evolutionary Computation, 1(1):3-17, 1997.

[46] V. Zivojnovic, S. Ritz, and H. Meyr. Multirate retiming: A powerful tool for hardware/software codesign. Technical report, Aachen University of Technology, 1993.

[47] V. Zivojnovic, S. Ritz, and H. Meyr. Retiming of DSP programs for optimum vectorization. In Proceedings of the International Conference on Acoustics, Speech, and Signal Processing, April 1994.

[48] W. Sung, J. Kim, and S. Ha. Memory efficient synthesis from dataflow graphs. In Proceedings of the International Symposium on Systems Synthesis, 1998.

[49] E. Zitzler, J. Teich, and S. S. Bhattacharyya. Multidimensional exploration of software implementations for DSP algorithms. Journal of VLSI Signal Processing Systems, 1999. Accepted for publication; to appear.

[50] Mentor Graphics Corporation. DSP Architect DFL User’s and Reference Manual, V 8.2_6. 1993.

[51] M. Levy. C compilers for DSPs flex their muscles. EDN Access, issue 12, June 1997. http://www.ednmag.com

[52] P. Paulin, M. Cornero, C. Liem, et al. Trends in Embedded Systems Technology. In: M.G. Sami, G. De Micheli (eds.): Hardware/Software Codesign, Kluwer Academic Publishers, 1996.

[53] K.M. Bischoff. Ox User's Manual. Technical Report \#92-31. Iowa State University, 1992. 
[54] A.V. Aho, R. Sethi, J.D. Ullman. Compilers - Principles, Techniques, and Tools. Addison-Wesley, 1986.

[55] G.J. Chaitin. Register Allocation and Spilling via Graph Coloring. ACM SIGPLAN Symp. on Compiler Construction, 1982, pp. 98-105.

[56] A.V. Aho, M. Ganapathi, S.W.K Tjiang. Code Generation Using Tree Matching and Dynamic Programming. ACM Trans. on Programming Languages and Systems, vol. 11, no. 4, 1989, pp. 491-516.

[57] C. Liem, T. May, P. Paulin. Instruction-Set Matching and Selection for DSP and ASIP Code Generation. European Design and Test Conference (ED \& TC), 1994, pp. 31-37.

[58] B. Wess. Automatic Instruction Code Generation based on Trellis Diagrams. IEEE Int. Symp. on Circuits and Systems (ISCAS), 1992, pp. 645-648.

[59] C.W. Fraser, D.R. Hanson, T.A. Proebsting. Engineering a Simple, Efficient Code Generator Generator. ACM Letters on Programming Languages and Systems vol. 1, no. 3, 1992, pp. 213-226.

[60] G. Araujo, S. Malik. Optimal Code Generation for Embedded Memory Non-Homogeneous Register Architectures. 8th Int. Symp. on System Synthesis (ISSS), 1995, pp. 36-41.

[61] S. Liao, S. Devadas, K. Keutzer, S. Tjiang, A. Wang. Code Optimization Techniques for Embedded DSP Microprocessors. 32nd Design Automation Conference (DAC), 1995, pp. 599-604.

[62] S. Liao, S. Devadas, K. Keutzer, S. Tjiang. Instruction Selection Using Binate Covering for Code Size Optimization. Int. Conf. on Computer-Aided Design (ICCAD), 1995, pp. 393-399.

[63] G. Araujo, S. Malik, M. Lee. Using Register Transfer Paths in Code Generation for Heterogeneous Memory-Register Architectures. 33rd Design Automation Conference (DAC), 1996

[64] D.J. Kolson, A. Nicolau, N. Dutt, K. Kennedy. Optimal Register Assignment for Loops for Embedded Code Generation. 8th Int. Symp. on System Synthesis (ISSS), 1995.

[65] A. Sudarsanam, S. Malik. Memory Bank and Register Allocation in Software Synthesis for ASIPs. Int. Conf. on Computer-Aided Design (ICCAD), 1995, pp. 388-392.

[66] D.H. Bartley. Optimizing Stack Frame Accesses for Processors with Restricted Addressing Modes. Software - Practice and Experience, vol. 22(2), 1992, pp. 101-110.

[67] S. Liao, S. Devadas, K. Keutzer, S. Tjiang, A. Wang. Storage Assignment to Decrease Code Size. ACM SIGPLAN Conference on Programming Language Design and Implementation (PLDI), 1995.

[68] R. Leupers, P. Marwedel. Algorithms for Address Assignment in DSP Code Generation. Int. Conf. on Computer-Aided Design (ICCAD), 1996.

[69] B. Wess, M. Gotschlich. Optimal DSP Memory Layout Generation as a Quadratic Assignment Problem. Int. Symp. on Circuits and Systems (ISCAS), 1997.

[70] A. Sudarsanam, S. Liao, S. Devadas. Analysis and Evaluation of Address Arithmetic Capabilities in Custom DSP Architectures. Design Automation Conference (DAC), 1997.

[71] R. Leupers, F. David. A Uniform Optimization Technique for Offset Assignment Problems. 11th Int. Symp. on System Synthesis (ISSS), 1998. 
[72] C. Liem, P.Paulin, A. Jerraya. Address Calculation for Retargetable Compilation and Exploration of Instruction-Set Architectures. 33rd Design Automation Conference (DAC), 1996.

[73] S. Davidson, D. Landskov, B.D. Shriver, P.W. Mallett. Some Experiments in Local Microcode Compaction for Horizontal Machines. IEEE Trans. on Computers, vol. 30, no. 7, 1981, pp. 460-477.

[74] A. Timmer, M. Strik, J. van Meerbergen, J. Jess. Conflict Modelling and Instruction Scheduling in Code Generation for In-House DSP Cores. 32nd Design Automation Conference (DAC), 1995, pp. 593-598.

[75] R. Leupers, P. Marwedel. Time-Constrained Code Compaction for DSPs. IEEE Trans. on VLSI Systems, Vol. 5, No. 1, 1997.

[76] M. Berkelaar. Eindhoven University of Technology. available at ftp.es.ele.tue.nl/pub/lp_solve/

[77] K. Rimey, P.N. Hilfinger. Lazy Data Routing and Greedy Scheduling for Application-Specific Signal Processors. 21st Annual Workshop on Microprogramming and Microarchitecture (MICRO-21), 1988, pp. 111-115.

[78] R. Hartmann. Combined Scheduling and Data Routing for Programmable ASIC Systems. European Conference on Design Automation (EDAC), 1992, pp. 486-490.

[79] T. Wilson, G. Grewal, B. Halley, D. Banerji. An Integrated Approach to Retargetable Code Generation. 7th Int. Symp. on High-Level Synthesis (HLSS), 1994, pp. 70-75.

[80] C.H. Gebotys. An Efficient Model for DSP Code Generation: Performance, Code Size, Estimated Energy. 10th Int. Symp. on System Synthesis (ISSS), 1997.

[81] S. Novack, A. Nicolau, N. Dutt. A Unified Code Generation Approach using Mutation Scheduling. Chapter 12 in [5].

[82] S. Bashford, R. Leupers. Constraint Driven Code Selection for Fixed-Point DSPs. 36th Design Automation Conference (DAC), 1999.

[83] R. Woudsma. EPICS: A Flexible Approach to Embedded DSP Cores. Int. Conf. on Signal Processing Applications and Technology (ICSPAT), 1994.

[84] R. Leupers. Retargetable Code Generation for Digital Signal Processors. Kluwer Academic Publishers, ISBN 0-79239958-7, 1997.

[85] R.M. Stallmann. Using and Porting GNU CC V2.4. Free Software Foundation, Cambridge/Massachusetts, 1993.

[86] L. Nowak. Graph based Retargetable Microcode Compilation in the MIMOLA Design System. 20th Ann. Workshop on Microprogramming (MICRO-20), 1987, pp. 126-132.

[87] D. Lanneer, J. Van Praet, A. Kifli, K. Schoofs, W. Geurts, F. Thoen, G. Goossens. CHESS: Retargetable Code Generation for Embedded DSP Processors. chapter 5 in [5].

[88] J. Van Praet, D. Lanneer, G. Goossens, W. Geurts, H. De Man. A Graph Based Processor Model for Retargetable Code Generation. European Design and Test Conference (ED \& TC), 1996.

[89] M.R. Hartoog, J.A. Rowson, P.D. Reddy, et al. Generation of Software Tools from Processor Descriptions for Hardware/Software Codesign. 34th Design Automation Conference (DAC), 1997.

[90] S. Hanono, S. Devadas. Instruction Selection, Resource Allocation, and Scheduling in the AVIV retargetable code generator. 35th Design Automation Conference (DAC), 1998. 
[91] G. Hadjiyiannis, S. Hanono, S. Devadas. ISDL: An Instruction-Set Description Language for Retargetability. 34th Design Automation Conference (DAC), 1997.

[92] ACE Associated Compiler Experts. http://www.ace.nl

[93] Target Compiler Technologies. http://www.retarget.com

[94] Archelon Inc. http://www.archelon.com 


\section{Biographical sketches of the authors}

Shuvra S. Bhattacharyya

Shuvra S. Bhattacharyya received the Ph.D. degree in Electrical Engineering and Computer Sciences from the University of California at Berkeley in 1994. Since July, 1997, he has been an Assistant Professor in the Department of Electrical and Computer Engineering at the University of Maryland at College Park. He holds a joint appointment with the University of Maryland Institute for Advanced Computer Studies (UMIACS).

Dr. Bhattacharyya's research interests center around computer-aided design for embedded systems, with emphasis on synthesis and optimization of hardware and software for digital signal/image/video processing (DSP) applications.

From 1991 to 1992, he was at Kuck and Associates, Inc. in Champaign, Illinois, where he was involved in the research and development of program transformations for performance improvement in C and Fortran compilers. From 1994 to 1997 , he was a Researcher at the Semiconductor Research Laboratory of Hitachi America, Ltd., in San Jose, California. At Hitachi, he was involved in research on software optimization techniques for embedded DSP applications.

Dr. Bhattacharyya is a recipient of the NSF CAREER award (1997), and is co-author of Software Synthesis from Dataflow Graphs (Kluwer Academic Publishers, 1996), and Embedded Multiprocessors: Scheduling and Synchronization (Marcel-Dekker, to be published in 2000).

\section{Rainer Leupers}

Rainer Leupers received his Diploma and Ph.D. degrees in Computer Science with distinction from the University of Dortmund, Germany, in 1992 and 1997, respectively. He received the Hans Uhde Award and the best dissertation award from the University of Dortmund for outstanding theses. Since 1993, he has been working as a researcher at the Computer Science Department at Dortmund, where he is currently heading the DSP compiler group. Dr. Leupers is the author of the book Retargetable Code Generation for Digital Signal Processors, published by Kluwer Academic publishers in 1997. His research interests include design automation and compilers for embedded systems.

\section{Peter Marwedel}

Peter Marwedel received his Ph.D. in Physics from the University of Kiel (Germany) in 1974. He worked at the Computer Science Department of that University from 1974 until 1989. In 1987, he received the Dr. habil. degree (a degree required for becoming a professor) for his work on high-level synthesis and retargetable code generation based on the hardware description language MIMOLA. Since 1989 he is a professor at the Computer Science Department of the University of Dortmund (Germany). He served as the Dean of that Department between 1992 and 1995. Currently, he is the president of the technology transfer institute ICD, located at Dortmund. His research areas include hardware/software codesign, high-level test generation, high-level synthesis and code generation for embedded processors. He is one of the editors of the book Code Generation for Embedded Processors published by Kluwer Academic publishers in 1995. Dr. Marwedel is a member of the IEEE Computer society, the ACM, and the Gesellschaft fur Informatik (GI). 